\title{
DATA REPORT OF OBLIQUE REFLECTION-REFRACTION RADIO- SONOBUOY PROFILES ON THE AFRICAN ATLANTIC CONTINENTAL MARGIN (R/V ATLANTIS II CRUISES 67 AND 75)
}

By

Hartley Hoskins, Carolyn U. Rogers, and Aileen 0 . Woo

WOODS HOLE OCEANOGRAPHIC INSTITUTION

Woods Hole, Massachusetts 02543

June 1974

\begin{abstract}
TECHNICAL REPORT
Prepared for the International Decade of ocean Exploration, National Science Foundation Grant GX-28193 for the Eastern Atlantic Continental Margin progrom.

Reproduction in whole or in part is permitted for ony purpose of the United States Government. In citing this manusaript in a bibliography, the reference should be followed by the phrase: UNPUBLISHED MANUSCRIPT.

Approved for public release; distribution un limited.
\end{abstract}

Approved for Distribution Seizoloth T. Bunces Elizabeth T. Bunce, Acting Chairman Department of Geology \& Geophysics 
TABLE OF CONTENTS

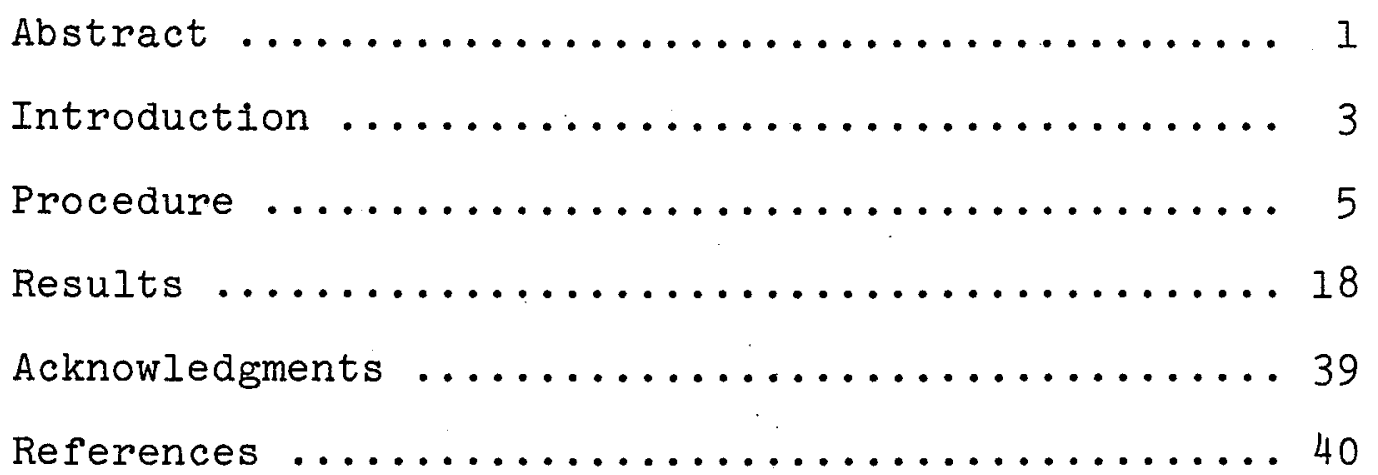


DATA REPORT OF OBLIQUE REFLECTION-REFRACTION RADIOSONOBUOY PROFILES ON THE AFRICAN ATLANTIC CONTINENTAL MARGIN

$$
\text { (R/V ATLANTIS II CRUISES } 67 \text { AND 75) }
$$

Hartley Hoskins, Carolyn U. Rogers, and Aileen O. Woo Woods Hole Oceanographic Institution, Woods Hole, Massachusetts 0,2543

$$
\begin{aligned}
& \text { ABSTRACT: Two hundred sixty-four unreversed oblique reflection- } \\
& \text { refraction profiles using expendable radio-sonobuoys were obtained } \\
& \text { during two geophysical cruises to the Atlantic continental margin } \\
& \text { of Africa. This data report gives the profile locations, a } \\
& \text { summary of the data collection and analysis, and } 780 \text { interval } \\
& \text { reflection and refraction velocities and thicknesses that were } \\
& \text { determined. }
\end{aligned}
$$




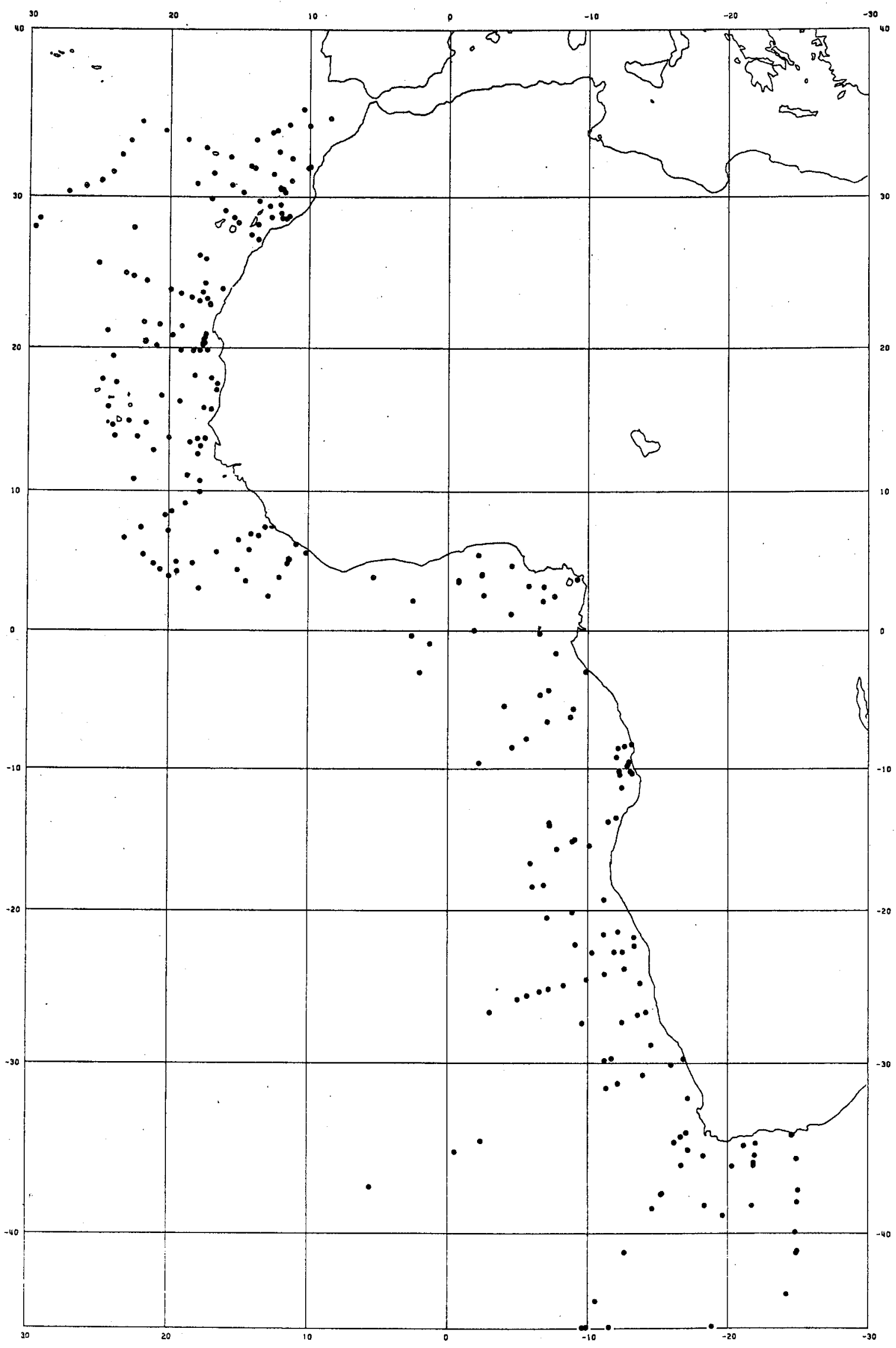

Figure 1. Geographic distribution of oblique reflection-refraction sonobuoy profile stations, R/V ATLANTIS II Cruises $67 \& 75$. 


\section{INTRODUCTION}

Two hundred sixty-four oblique reflection-refraction profiles using expendable radio-sonobuoys were taken during the Eastern Atlantic Continental Margin program sponsored by the International Decade of Ocean Exploration (Fig. 1). The program covering 95,800 line kilometers, was carried out during cruises 67 (1972) and 75 (1973) of R/V ATLANTIS II of the woods Hole Oceanographic Institution. About a third of the sonobuoy profiles were processed at sea.

The sonobuoys, modified military type AN/SSQ-41, were purchased from Oceanology International of college Station, Texas. Each broadcasted at one of 16 frequency allocations in the $162 \mathrm{MHz}$ band and had a hydrophone suspended on a compliant cable at either 19 meters (60 feet) or 91 meters (300 feet) below the buoy. In nearly all deployments the 19-meter depth was used to keep the hydrophone within the wave-mixed, uniform temperature, surface layer enabling better detection of the direct water wave arrival.

The ship steamed away from the buoys at 7 to $9 \mathrm{~km} / \mathrm{hour}$ and the source repetition rate was between 10 and 13 seconds -- 20 to $30 \mathrm{~m}$ travel between shots. The slow ship speed was chosen to keep the travel time step-out of the reflected wavetrains between successive shots small enough to maintain good visual coherence on the recording. 
The sources were a 2.0 liter (120 cu. in.) and/or a 4.9 liter (300 cu. in.) Bolt PAR airgun operated at a pressure of about $1.2 \times 10^{7} \mathrm{~N} / \mathrm{m}^{2}$ (1700 psi). The reflected and refracted returns were displayed on a graphic recording using a 7.5 or 8.0 second sweep commencing shortly before the onset of the seafloor reflection. The useful range of the reflection traces was generally limited to three times the normal-incidence travel time because of the convergence of the returns and the decrease in. intensity of the returns from buried horizons. Radio range of the buoy was about 20 seconds water wave travel time $(30 \mathrm{~km})$.

The data were concurrently recorded on a 4-track Tandberg Series 100 FM tape recorder. The channel allocations were: 1) normal incidence reflection profile detected with the towed streamer, 2) tape speed compensation signal, 3) oblique reflectionrefraction return detected by the sonobuoy, and 4) voice announcements and shot instant. At $17 / 8$ inches per second recording speed the bandpass is $0.1-313 \mathrm{~Hz}$.

A bandpass filter of 15 to $35 \mathrm{~Hz}$ provided the most discernible display of the reflection data. The direct water wave arrival was often difficult to follow at range. Thus, determination of the travel time from the ship-towed source to the sonobuoy hydrophone was the poorest part of the measurement. At times, a replay of the tape at a higher filter bandpass was effective in better detection of the direct water wave travel time. 


\section{PROCEDURE}

Terrains of flat or uniformly sloping horizons are best suited for oblique reflection-refraction profile determinations because the computation used is based on rectilinear propagation of rays through layers of constant velocity separated by planar interfaces. In a majority of launchings, we were successful in anticipating a favorable attitude of the reflecting horizons over which the ship subsequently passed. Compressional wave velocities were only determined where the reflecting interfaces had a lateral extent of a few kilometers and a slope of less than 5 degrees as measured on the normal-incidence profile.

The velocity determination is very sensitive to accurate travel time measurements. Our practice was to trace the most continuous cycle rather than the leading edge of the reflection wavelet. The minimum increment in normal-incidence travel time between layers was chosen to be at least 0.2 seconds because of: 1) the duration of the airgun pulse $(0.2$ sec.), and 2) the deviations of the picks from the best fitted line having to be smaller than the thickness of the layer.

A data sheet (Fig. 2) detailing pertinent information about each sonobuoy was prepared. Since the determination of range to the buoy from the ship-towed source could vary as much as $5 \%$, redundancy was sought by comparing two or more measurements of the shallow water temperature and the ship's speed. Because all 


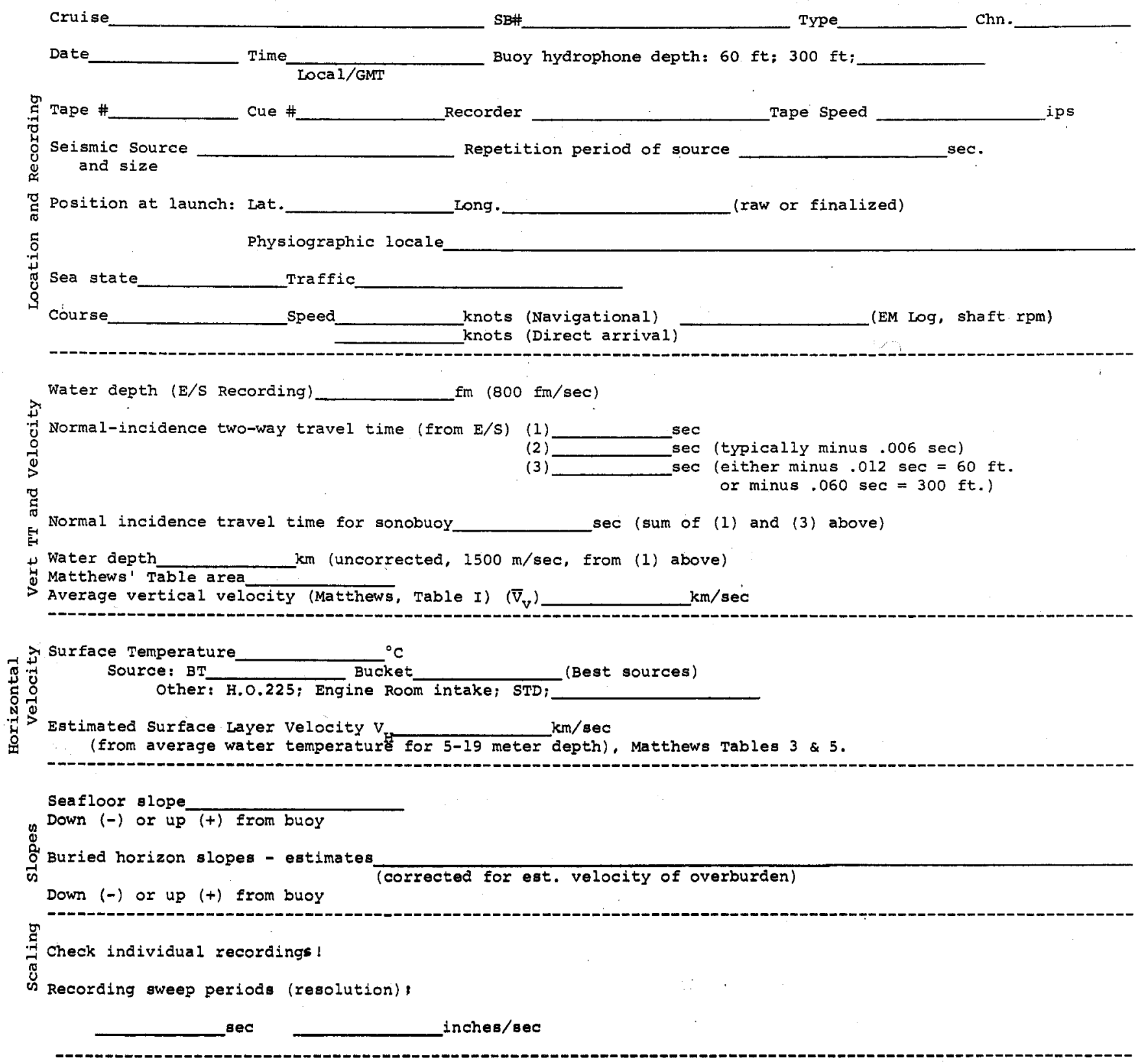

Comments:

Sonobuoy data sheet: Rev. $6 / 74$ 
of the profiles are unreversed, the slopes of the horizons were estimated from the concurrent normal-incidence recording. The slopes were corrected for the vertical exaggeration of the recording due to ship's speed, recorder sweep period and advance, and source repetition rate (Fig. 3), and for the compressional wave velocity of the overburden (Fig. 4).

Those reflecting horizons that were prominent and extensive on the concurrent normal-incidence recording were selected, where possible, on the sonobuoy profiles. These reflection sequences and refractions arrivals were traced on a transparent overlay with a muted grid of 10 divisions to the inch. The useable range of the reflection trace is limited by the significant deviation that occurs in water from straight-line propagation of rays at angles greater than $45^{\circ}$ from the normal at the sea surface. The direct, reflection, and refraction arrivals were read at equal travel time intervals. A range-travel time plot based on the information from the data sheet and the digitized data was made using program PORP (Plot oblique Reflection Profiles) (Fig. 5). Edits were made where necessary. The best line was fitted to the squares of the ranges and travel times for the reflections, and to the ranges and travel times for the refractions using program LINFT (IINear FiT) (Fig. 6). The tracing and the accuracy of the digitization was checked by the deviations from the best fitting lines. Then the reflection data were processed by 


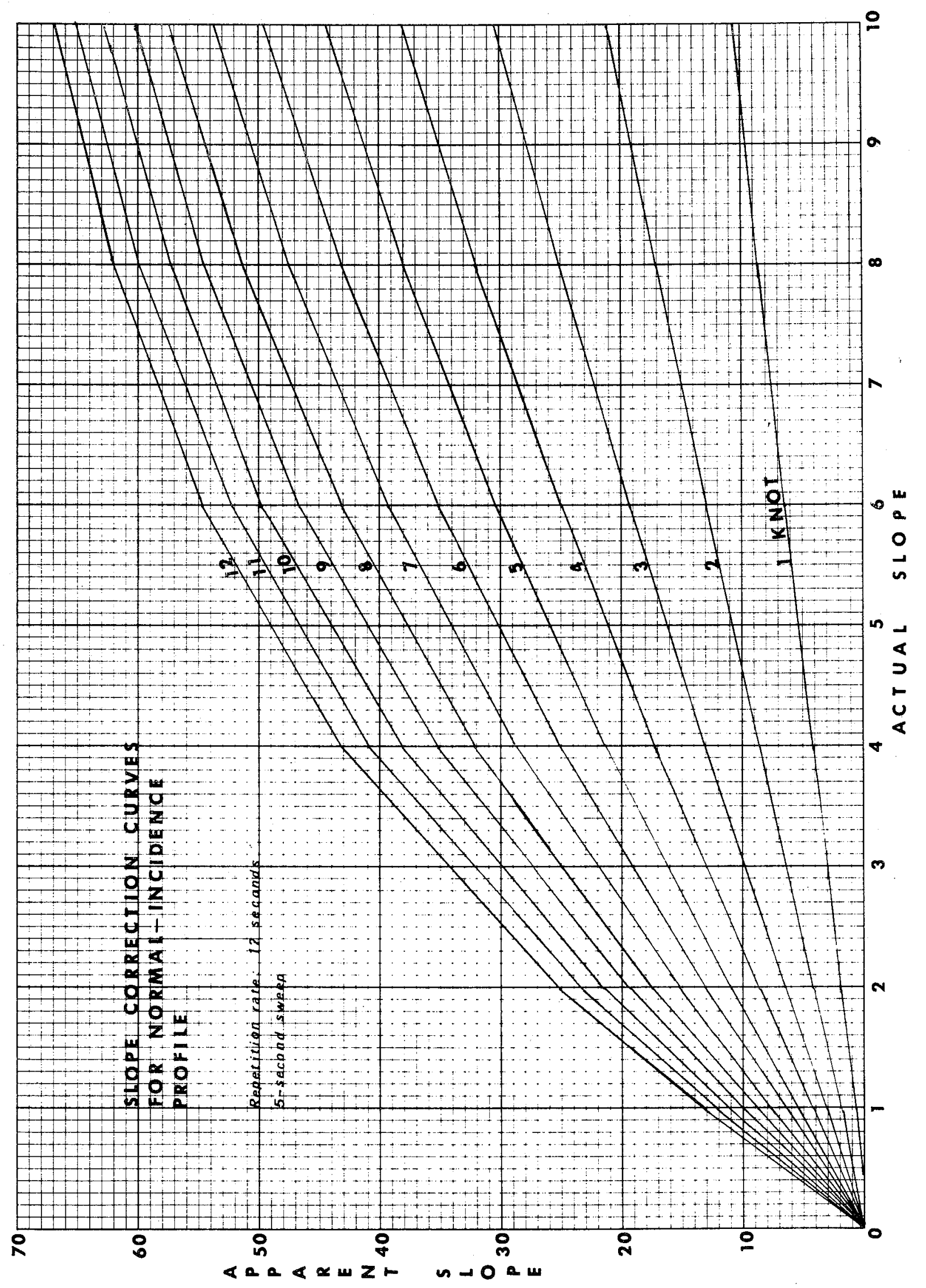

Figure 3. Sample slope correction curves for normal-incidence profile on SDA7003 10-inch graphic recorder. 


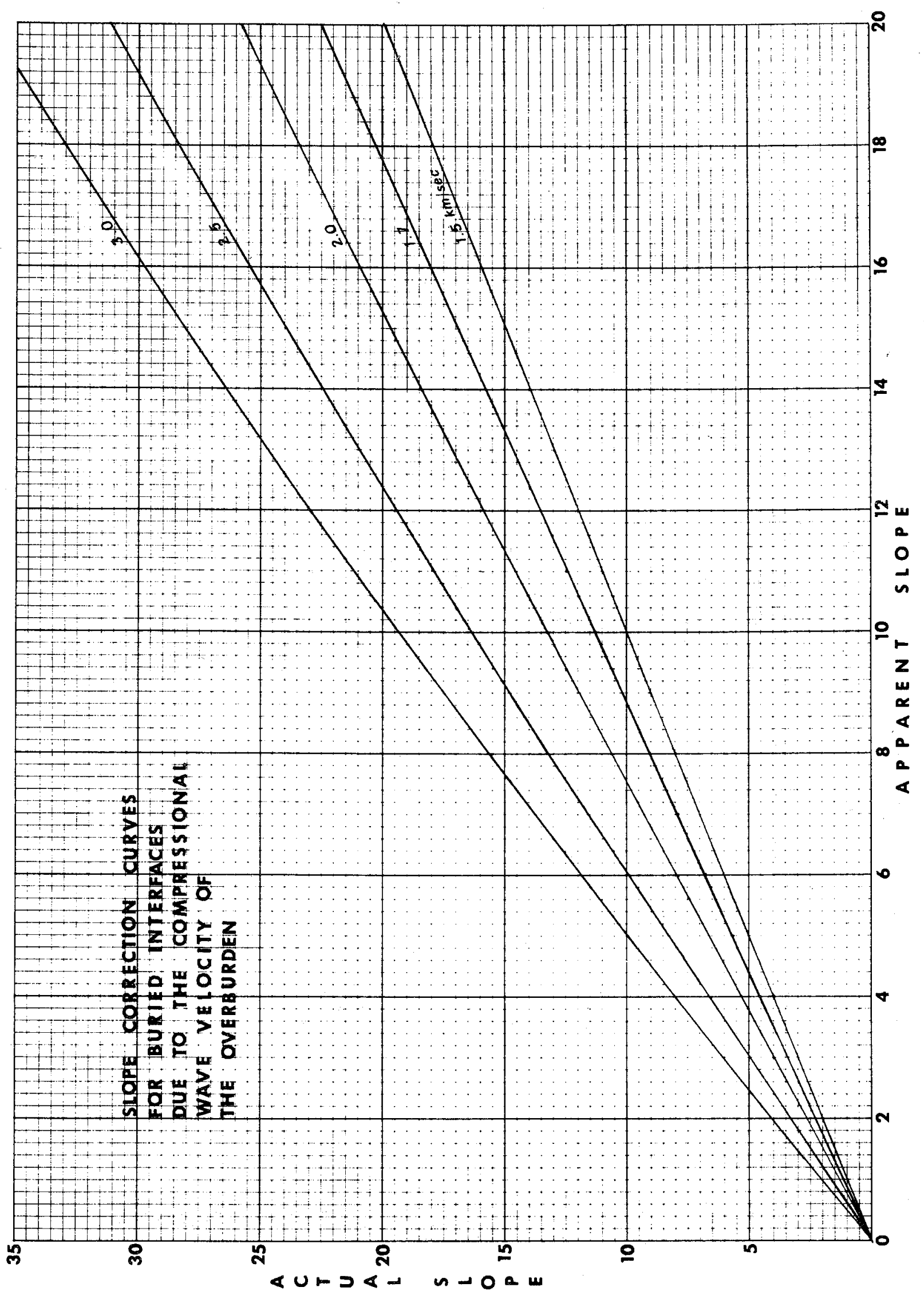

Figure 4. Slope correction curves for buried interfaces due to the compressional wave velocity of the overburden. 


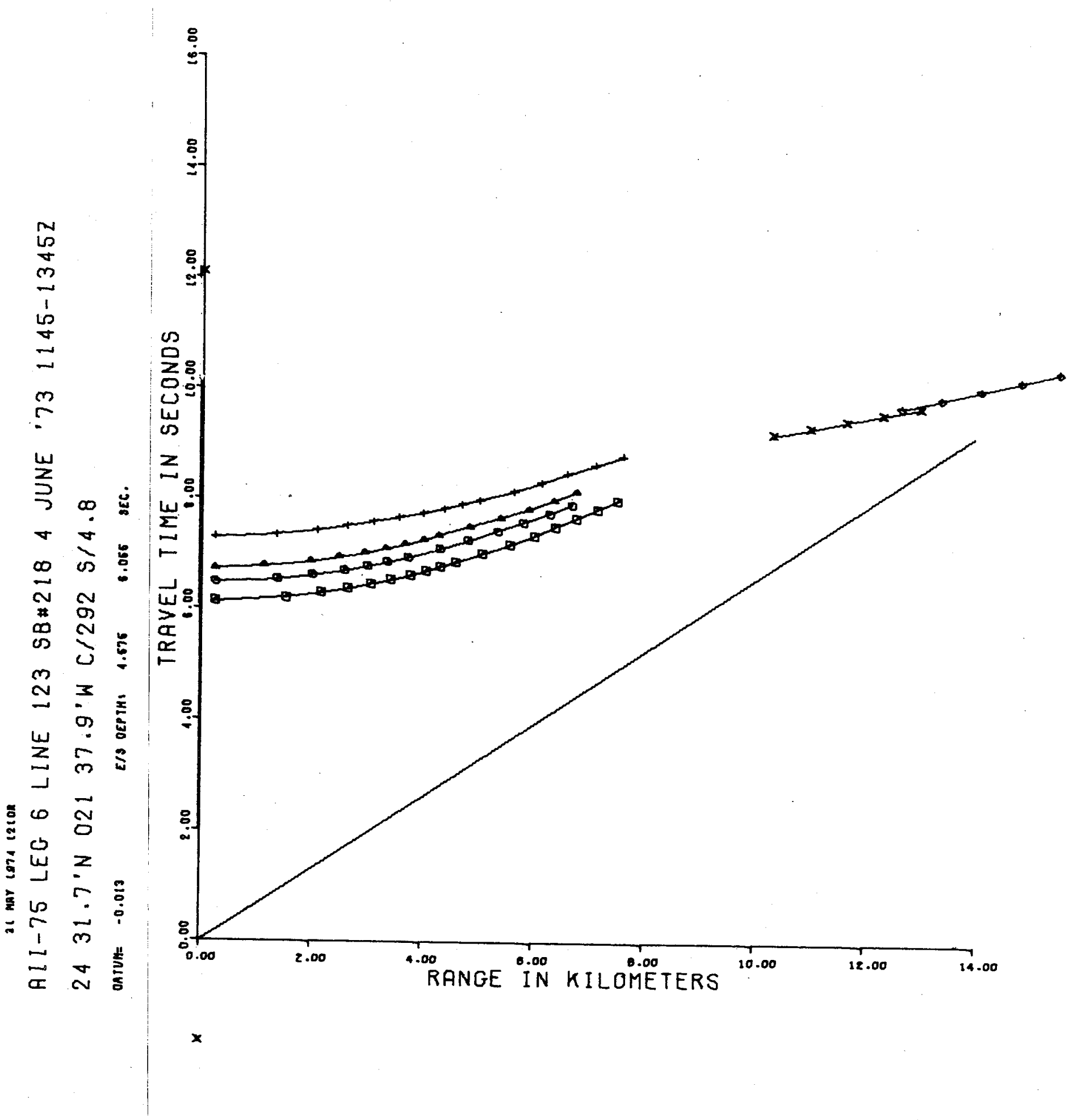

Figure 5. Sample range-travel time plot of the digitized data using Program PORP. 


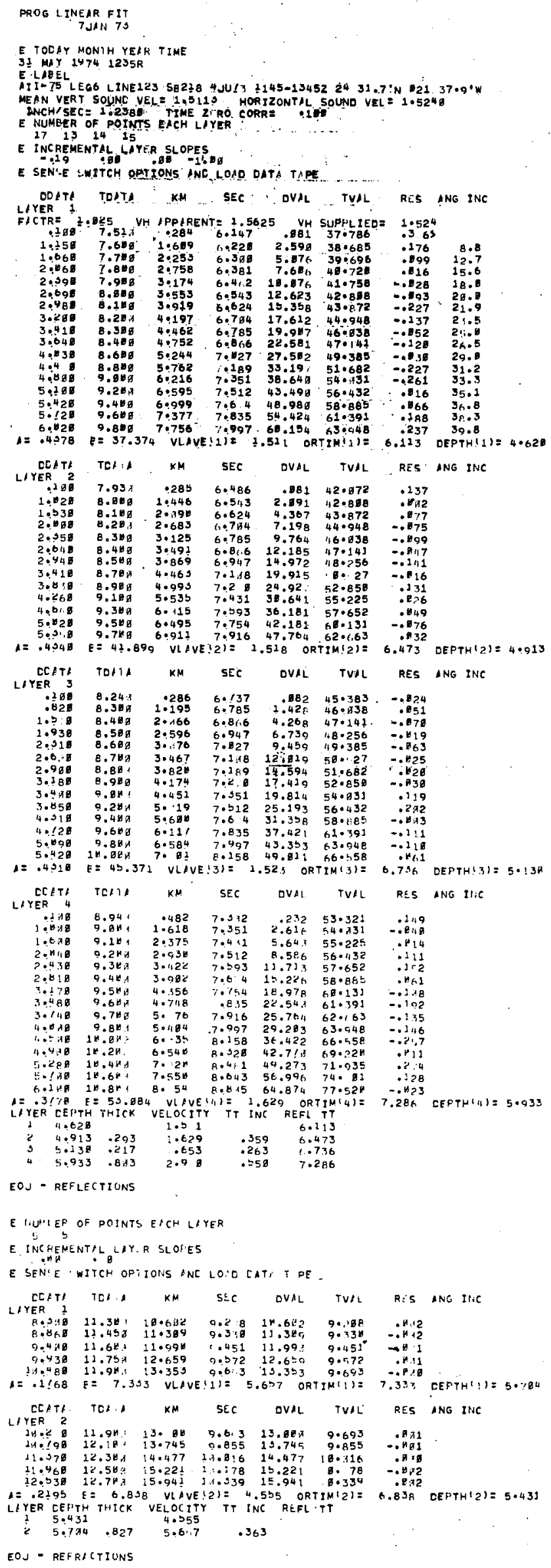

Figure 6. Sample listing of LINFT computations. 


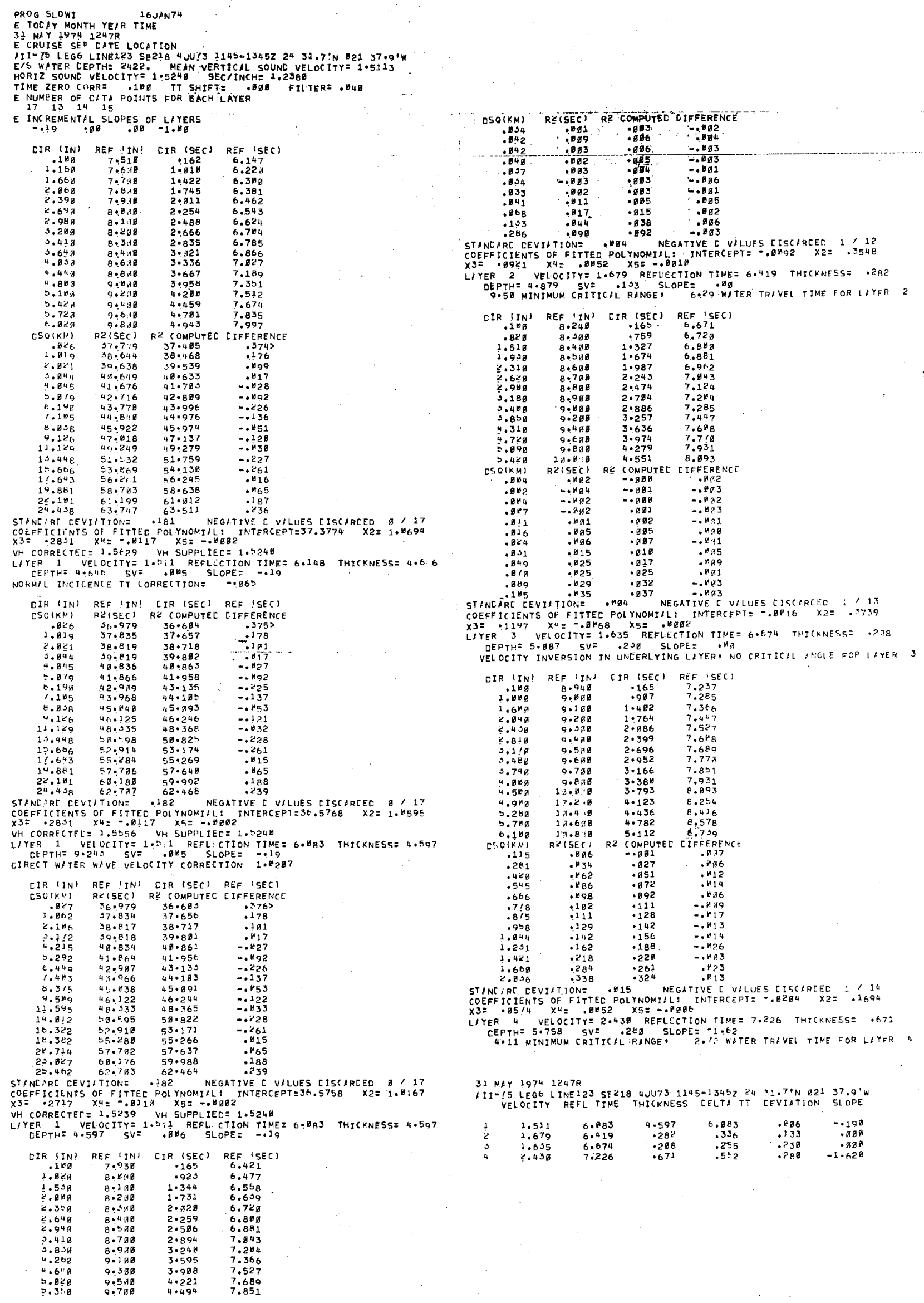

Figure 7. Sample listing of SLOWI computations 
program SLOWI (SLOping WIde angle reflection profiles) (Fig. 7) (Le Pichon, et al., 1968, Houtz, et aㅡ., 1968) which: 1) adjusts the direct water wave arrival times to match one predicted from the seafloor reflection based on the normal-incidence travel time, the seafloor slope, and good estimates of horizontal and mean vertical sound velocities, 2) fits the oblique reflection trace with a fourth-order polynomial which when differentiated determines the emergent angle of the wavefront at the sea surface as a function of range, 3) computes the reduced ranges and travel times for each layer knowing the emergent angle, the normal-incidence travel time for the water and successive layers, and the slopes of the horizons, and 4) fits a straight line to the squares of the reduced ranges and travel times for each layer to determine the velocity and thickness.

Where possible, each refraction was matched to its corresponding oblique reflection. A simple modeling program which computes and plots expected travel times versus ranges helped verify some of the interpretations.

Four examples of the sonobuoy profiles obtained from different physiographic areas are shown in Figs. 8, 9, 10, and 11. Each figure shows the sonobuoy profile, its tracing, and the concurrent normal incidence reflection profile with its interpretation. The symbols used in the figures are: $D$, the direct water wave arrival, and $V$, the computed velocity. 

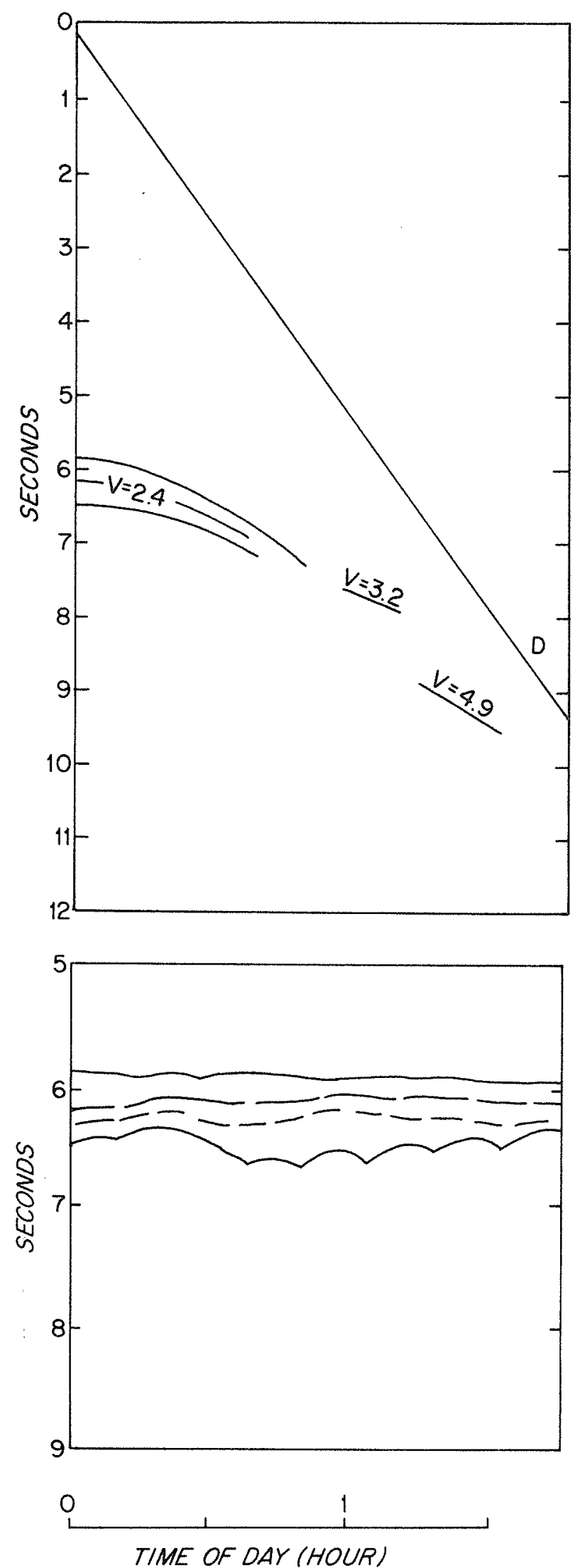
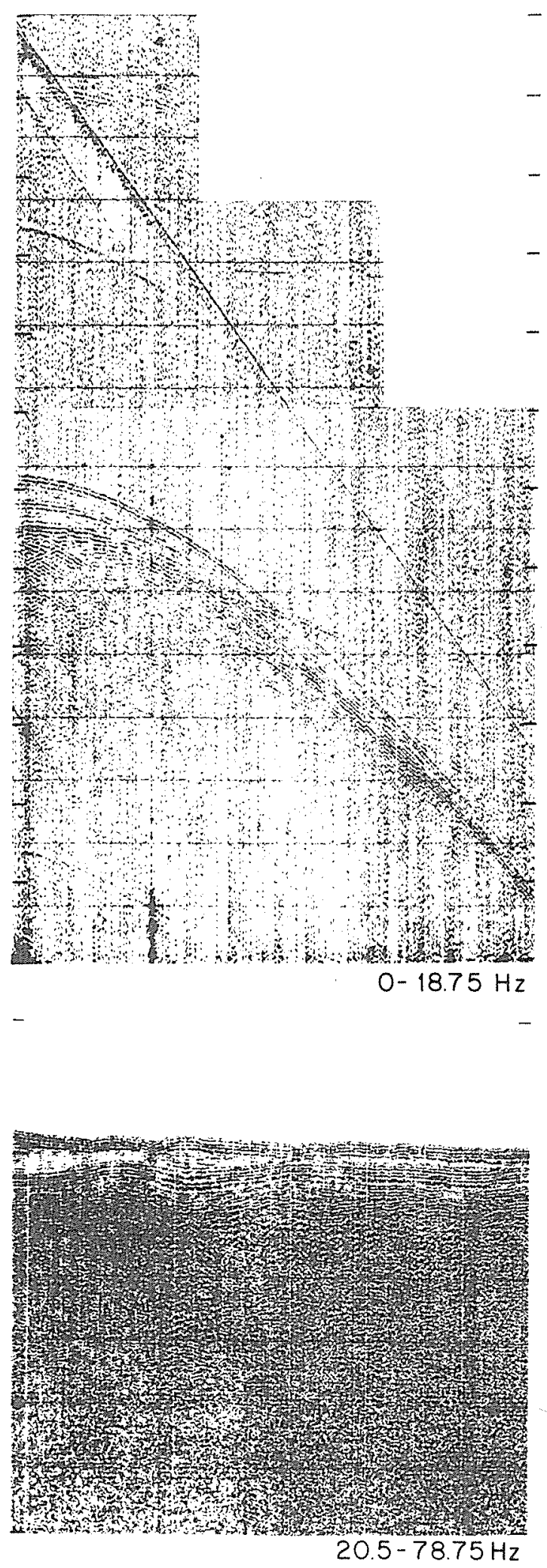

SB 11

Figure 8. Sonobuoy and concurrent normal-incidence profile obtained from the southwest Agulhas Basin, north of the Atlantic Indian Ridge. This profile shows a thin sediment layer over shallow oceanic basement. The velocity in the sediments was computed using the oblique reflections. Deeper velocities were determined from the refractions. 

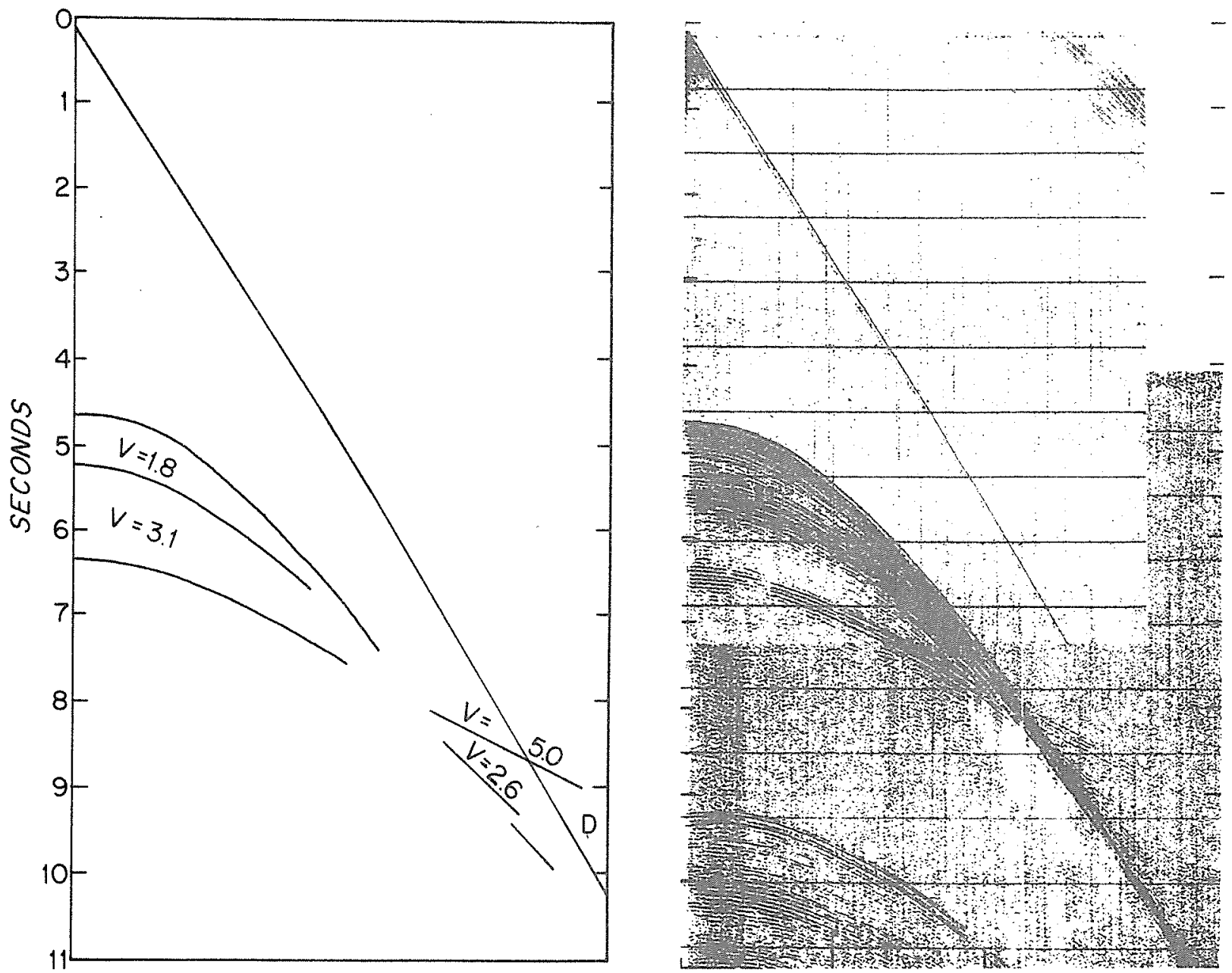

$15-50 \mathrm{~Hz}$
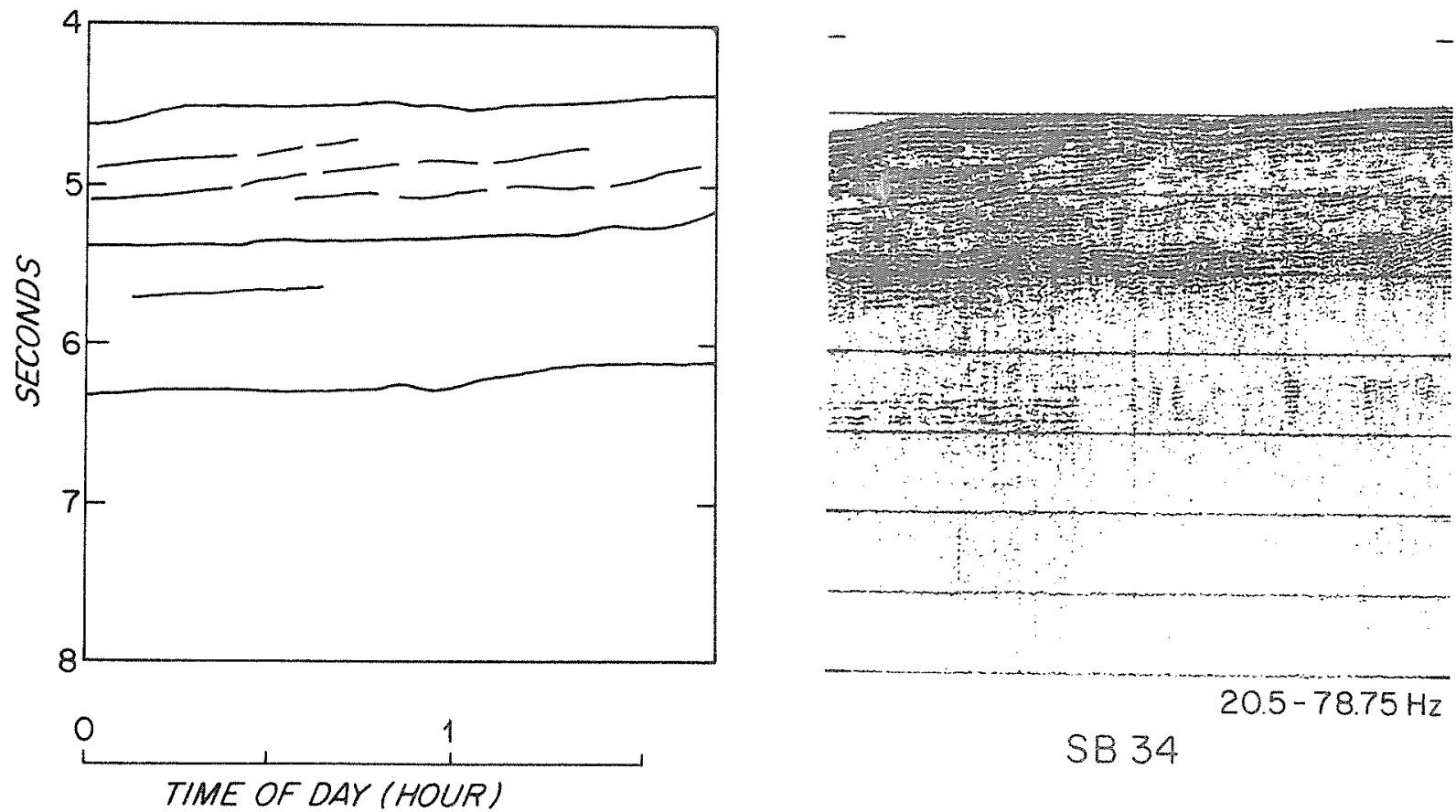

SB 34

Figure 9. Sonobuoy and concurrent normal incidence profiles obtained from the continental rise west of Cape Town. These profiles show strong discrete horizons. The velocity in the shallow sediments were computed from the oblique reflections and that the deeper sedimentary horizon and basement from the refraction returns. 

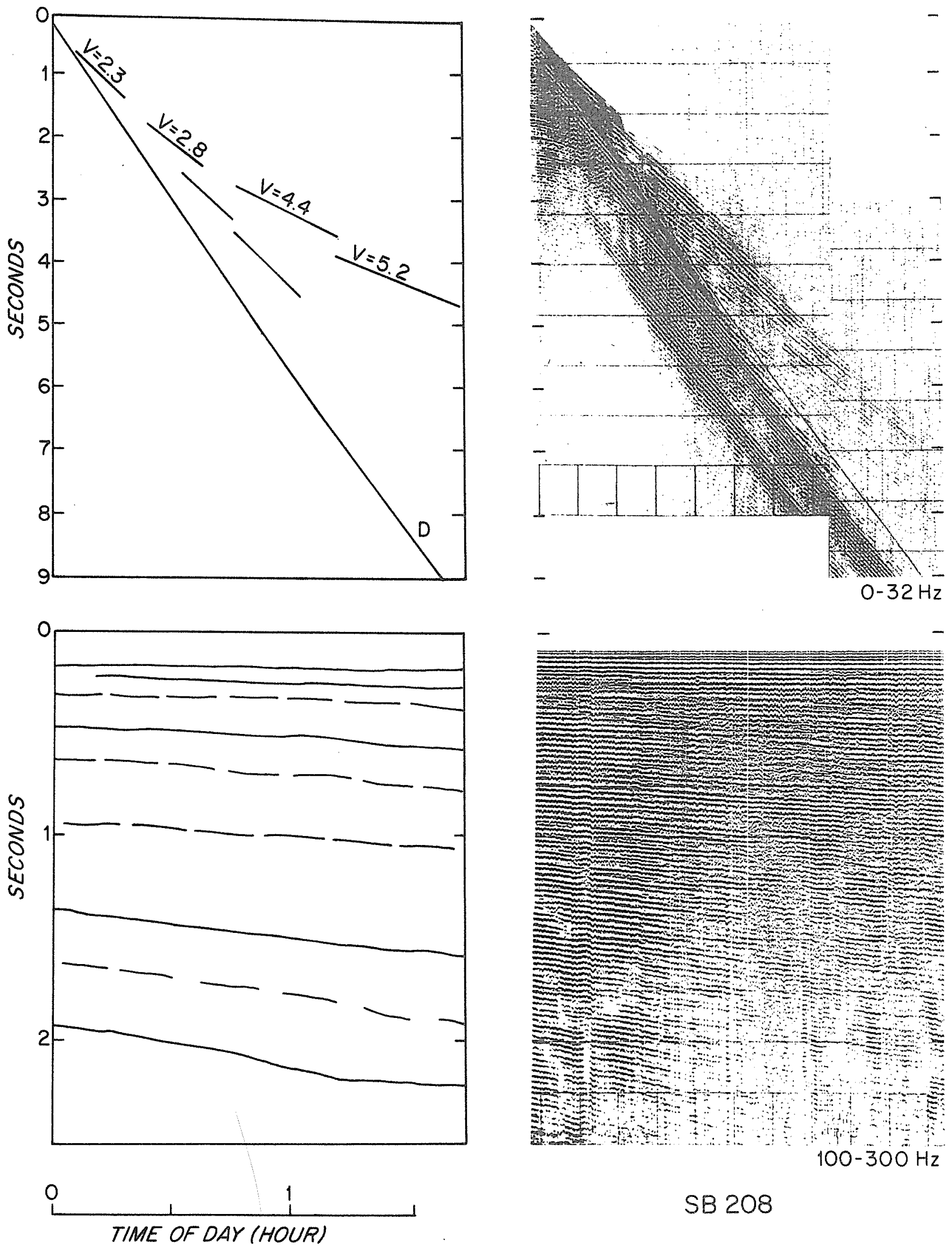

SB 208

Figure 10. Sonobuoy and concurrent normal incidence profile obtained from the continental shelf off Spanish Sahara and Mauritania. Only refraction returns were analyzed due to the shallowness of the water and the strong interference of the multiple water-column arrivals with the reflections from deeper interfaces. These velocity and depth determinations were used to identify horizons on the normal-incidence profile. 

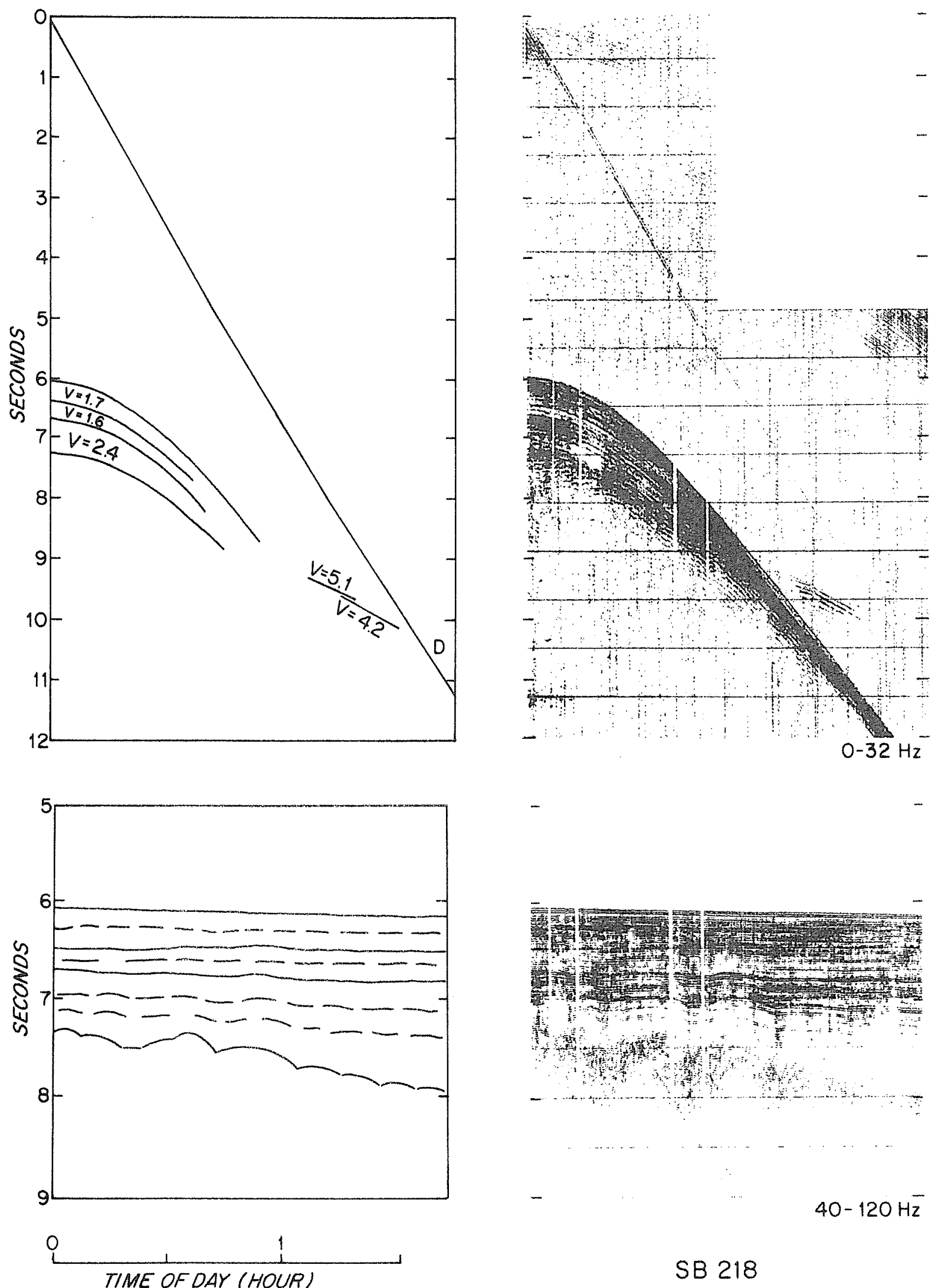

SB 218

Figure 11. Sonobuoy and concurrent normal-incidence profile from the southeast Canary Basin, southwest of the Canary Islands. This profile shows a thick sedimentary layer over the basement typical of an abyssal plain. Oblique reflections were used to compute horizons within the sediments while the basement velocity was determined from the refractions. 
A more complete description of the data collection and analytical procedures may be found in Knott and Hoskins, A Guide for the Collection and Analysis of Seismic Refraction and Oblique Reflection Data Received by Sonobuoys, in preparation.

\section{RESULTS}

The following chronological tabulation was prepared from a card file of layer determinations made for each oblique reflection-refraction sonobuoy profile. Symbols used in this tabulation are:

+ layer velocity determined from refractions

C layer velocity determined from critical seafloor reflection

* estimated velocity

? questionable determination

1 basement

A positive dip indicates that the horizon is shoaling in respect to the sea surface along the course from the sonobuoy. 
$-19-$

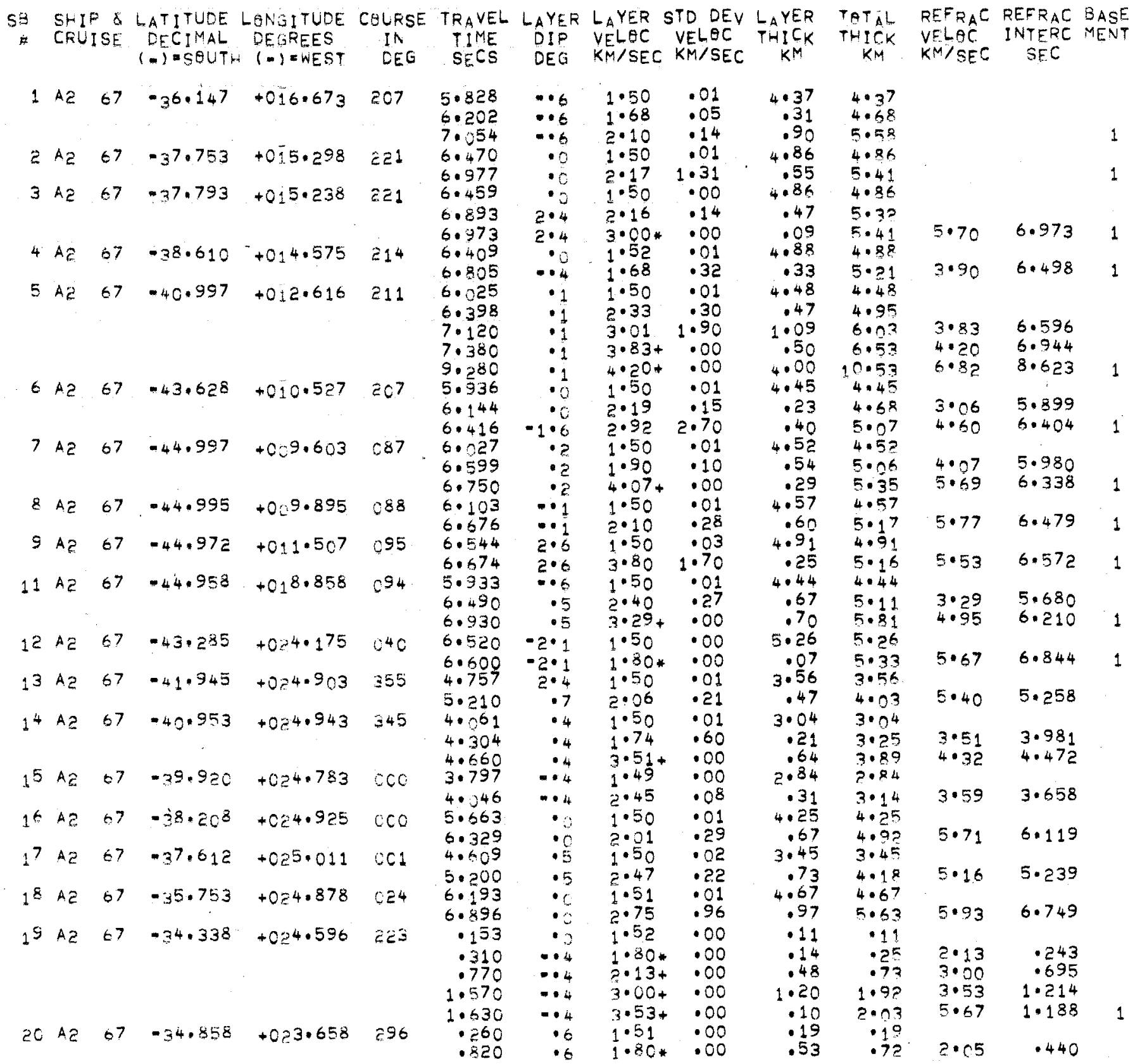




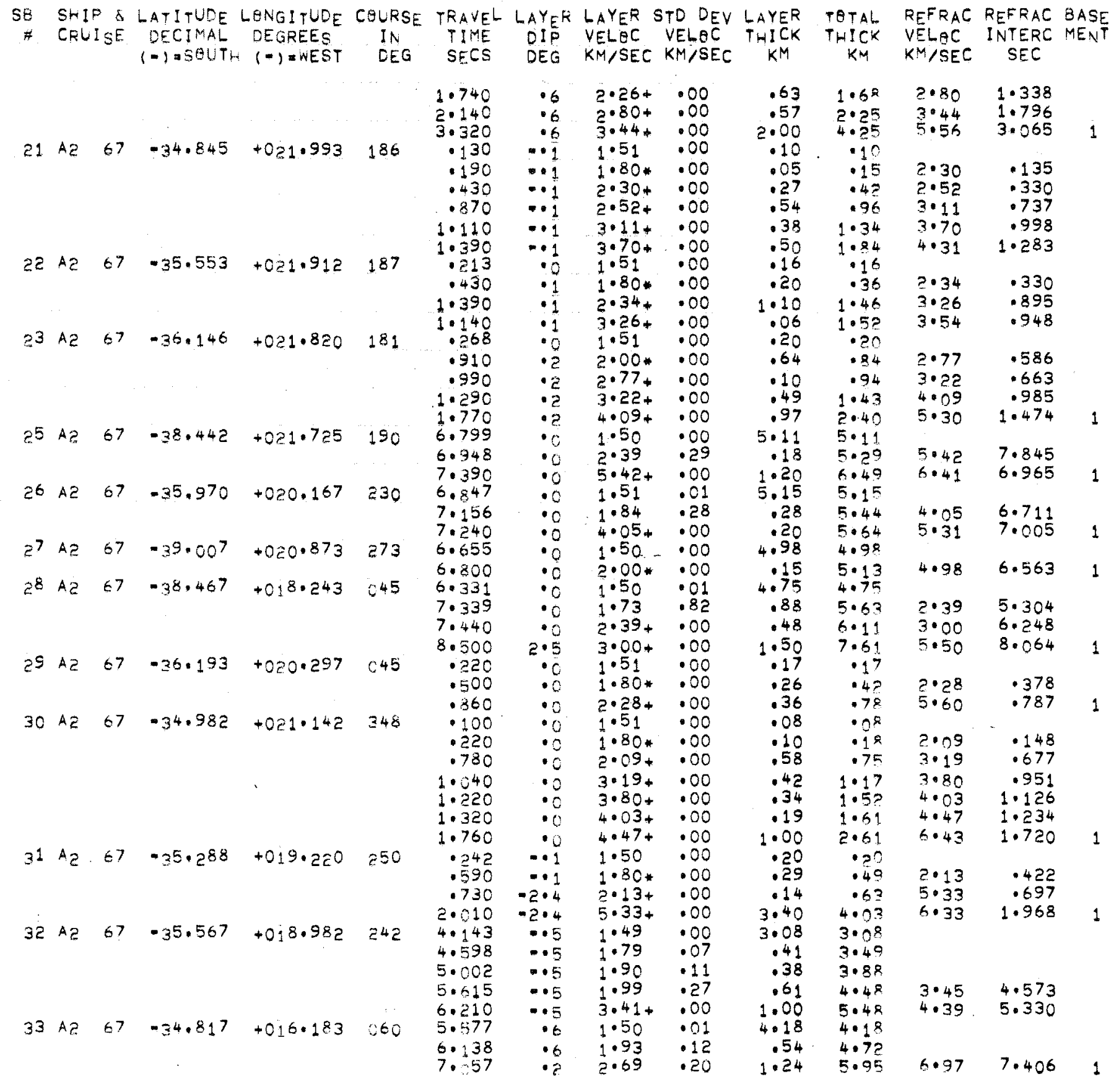


SB SHIP I LATITUDE LENGITUDE CBLRSE TRAVEL LAYER LAYER STD DEV LAYER H CRUISE OECIMAL DEGREES IN TIME $(-)$ aSQUTH $(-)=$ WEST CEQ

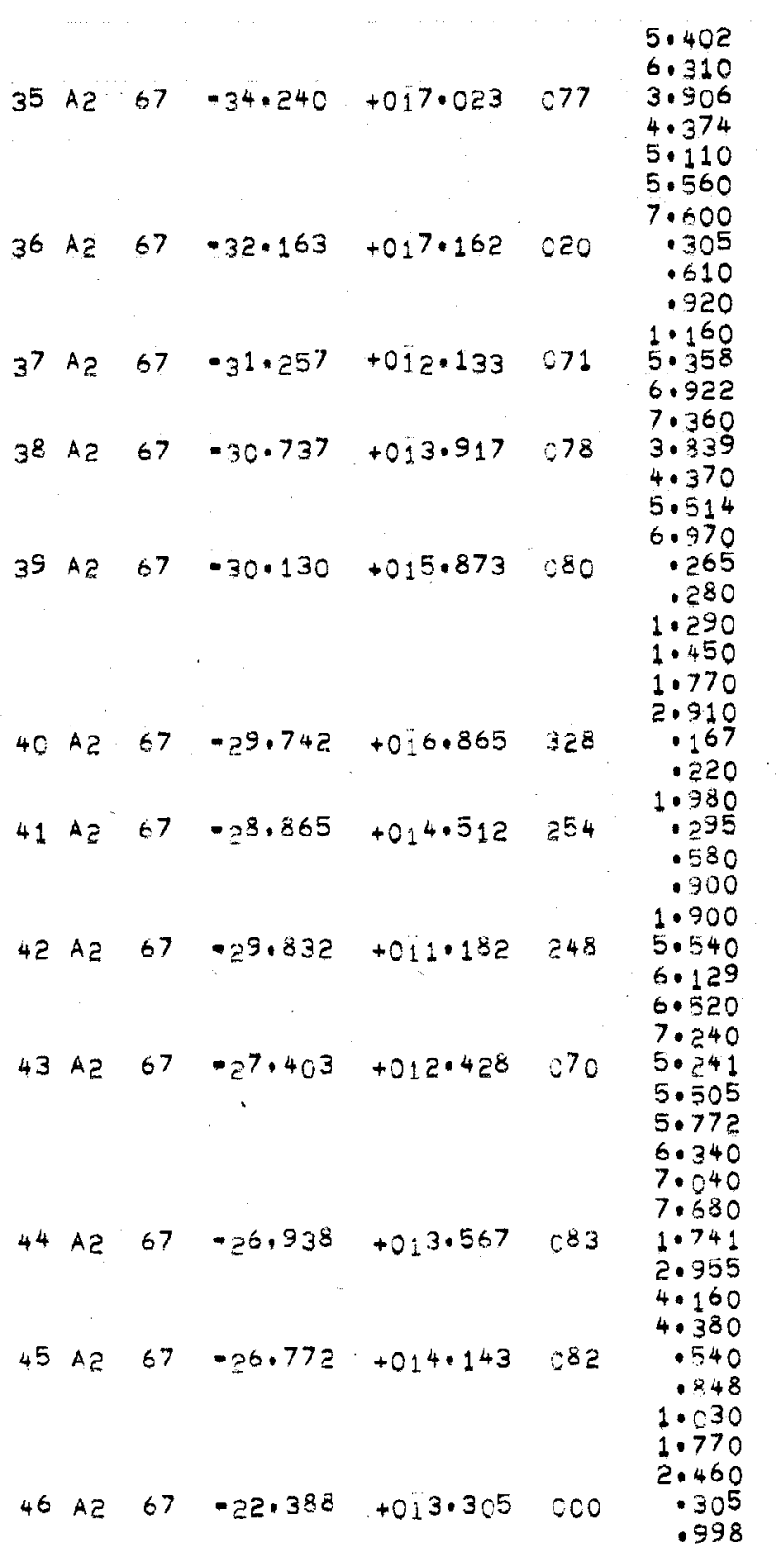

TETAL. DIF VELOC VELOC THICK DEG KM/SEC KM/SEC KM

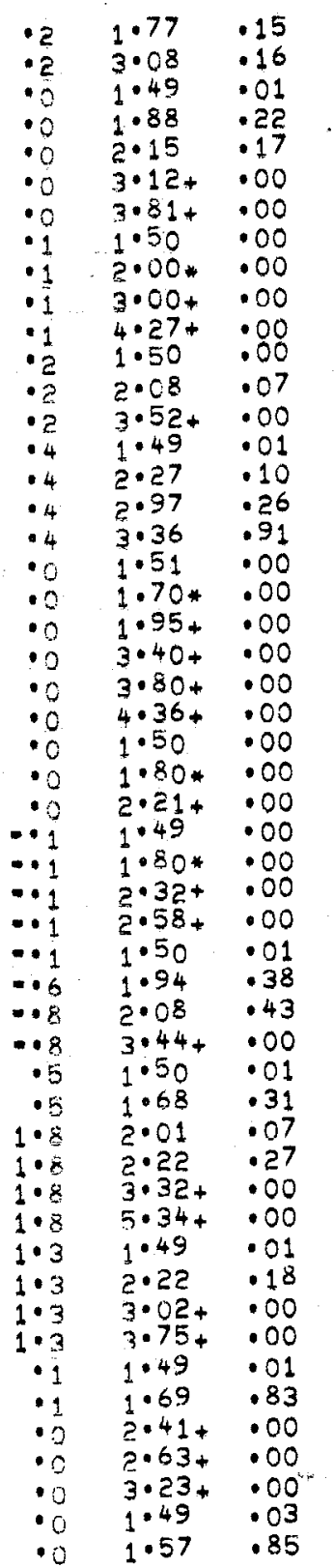

.69
1.40
2.91
.44
.79
.70
4.00
.23
.31
.47
.51
4.01
1.62
.77
2.86
.60
1.70
2.45
.20
.02
.96
.28
.60
.25
.13
.05
1.94
.22
.25
.37
1.26
4.15
.57
.41
1.30
3.92
.22
.27
.63
1.20
1.70
1.30
1.35
1.80
.40
.40
.26
.21
.96
1.10
.22
.54
1.90

REFRAC REFRAC BASE VELAC INTERC MENT KMISEC SEC
$2.64 \quad 4.485$ $5.02 \quad 6.065$

5.53 $2 \cdot 91$
$3 \cdot 35$ $4 \cdot 14$ $4 \cdot 84$ $8 \cdot 84$ $\cdot 23$ .54 1.01

$1 \cdot 52$ 4.01

$5 \cdot 64$

6.41 $2 \cdot 86$ 3.46

5.16

$5 \cdot 16$
7.61

.20 . 2 ?

$1 \cdot 18$

$1 \cdot 46$ $2 \cdot 06$ $2 \cdot 31$ .13 2.12

$2 \cdot 12$

.46

.83

$2 \cdot 09$

$4 \cdot 15$

$4 \cdot 7$ ?

$5 \cdot 13$

$6 \cdot 43$

$3 \cdot 9$ ?

$4 \cdot 14$

4.73

5.04

$3 \cdot 24$
7.94

$7 \cdot 94$

$1 \cdot 30$
2.64

$2 \cdot 64$

$4 \cdot 84$

.40

.66
.87

- 87

0.83
1.93

$5 \cdot 93$

.76

\begin{tabular}{|c|c|}
\hline $\begin{array}{l}3 \cdot 12 \\
3 \cdot 81 \\
7 \cdot 16\end{array}$ & $\begin{array}{l}4.290 \\
4.916 \\
7.276\end{array}$ \\
\hline $\begin{array}{l}3 \cdot 00 \\
4 \cdot 27 \\
6 \cdot 50\end{array}$ & $\begin{array}{r}.562 \\
.902 \\
1.165\end{array}$ \\
\hline $\begin{array}{l}3 \cdot 52 \\
5 \cdot 50\end{array}$ & $\begin{array}{l}6.159 \\
6.974\end{array}$ \\
\hline $\begin{array}{l}3 \cdot 32 \\
6 \cdot 00\end{array}$ & $\begin{array}{l}4.645 \\
6.412\end{array}$ \\
\hline $\begin{array}{l}1 \cdot 95 \\
3 \cdot 40 \\
3 \cdot 80 \\
4 \cdot 36 \\
5 \cdot 80\end{array}$ & $\begin{array}{r}.853 \\
1.170 \\
1.350 \\
1.676 \\
2.841\end{array}$ \\
\hline $\begin{array}{l}2 \cdot 21 \\
4 \cdot 00\end{array}$ & $\begin{array}{r}168 \\
1.646\end{array}$ \\
\hline $\begin{array}{l}2 \cdot 32 \\
2 \cdot 58 \\
3 \cdot 30\end{array}$ & $\begin{array}{r}.438 \\
.737 \\
1.688\end{array}$ \\
\hline $\begin{array}{l}3 \cdot 44 \\
6 \cdot 00\end{array}$ & $\begin{array}{l}5.794 \\
6.888\end{array}$ \\
\hline $\begin{array}{l}3 \cdot 32 \\
5 \cdot 34 \\
7 \cdot 30\end{array}$ & $\begin{array}{l}5.575 \\
6.606 \\
7.388\end{array}$ \\
\hline $\begin{array}{l}3 \cdot 02 \\
3 \cdot 75 \\
4 \cdot 77\end{array}$ & $\begin{array}{l}2.379 \\
3.620 \\
3.974\end{array}$ \\
\hline $\begin{array}{l}2 \cdot 41 \\
2 \cdot 63 \\
3 \cdot 23 \\
4 \cdot 22\end{array}$ & $\begin{array}{r}.722 \\
.900 \\
1.643 \\
2.392\end{array}$ \\
\hline $2 \cdot 34$ & .588 \\
\hline
\end{tabular}

R/V ATLANTIS II 67 AND 75 
$-22-$

SB SHIP \& LATITUDE LONGITUDE CQURSE TRAVEL LAYER LAYER STD DEV LAYER CRUISE DECIMAL DEGREES IN TIME (-) =SOUTH (-) \&WEST DEG SECS DIP VELOC VELOE THICK DE

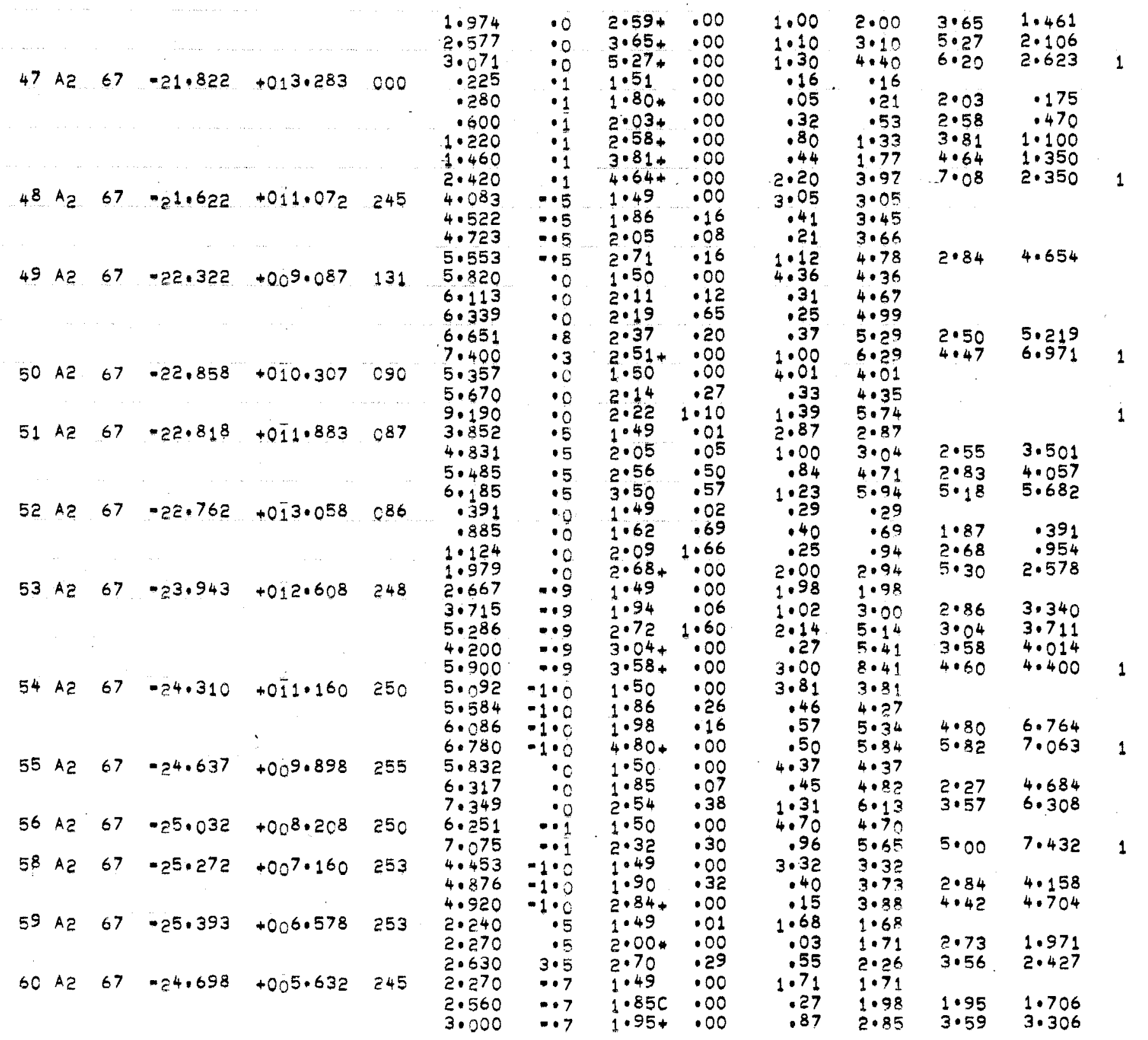

$F A G E \quad 4$

TOTAL REFRAC REFRAC BASE HICK VELOC INTERC MENT 
SB SHIF L LATITUDE LONGITUDE CQLRSE TRAVEL LAYER LAYER STD DEY LAYER $\#$ $(-)=$ SQUTH $(=)$ WEST
IN DEG
LAYER LAY

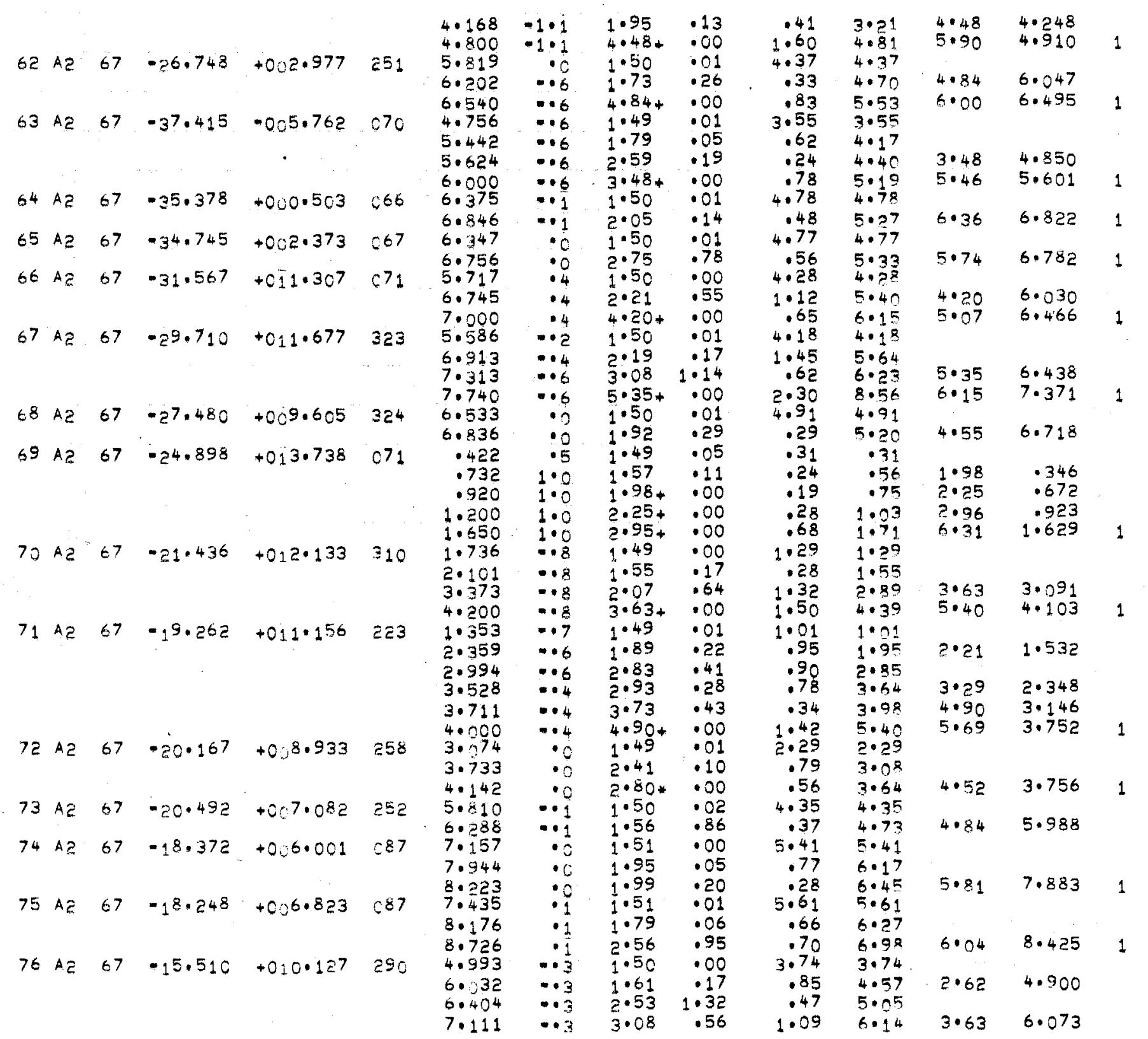

TATAL

THICK KM
REFRAC REFRAC BASE VELAC INTERC MENT KMISEC SEC 
SB SHIF \& LATITUDE LQNGITUDE COLRSE TRAVEL LAYER LAYER STD DEV LAYER TOTAL

SB

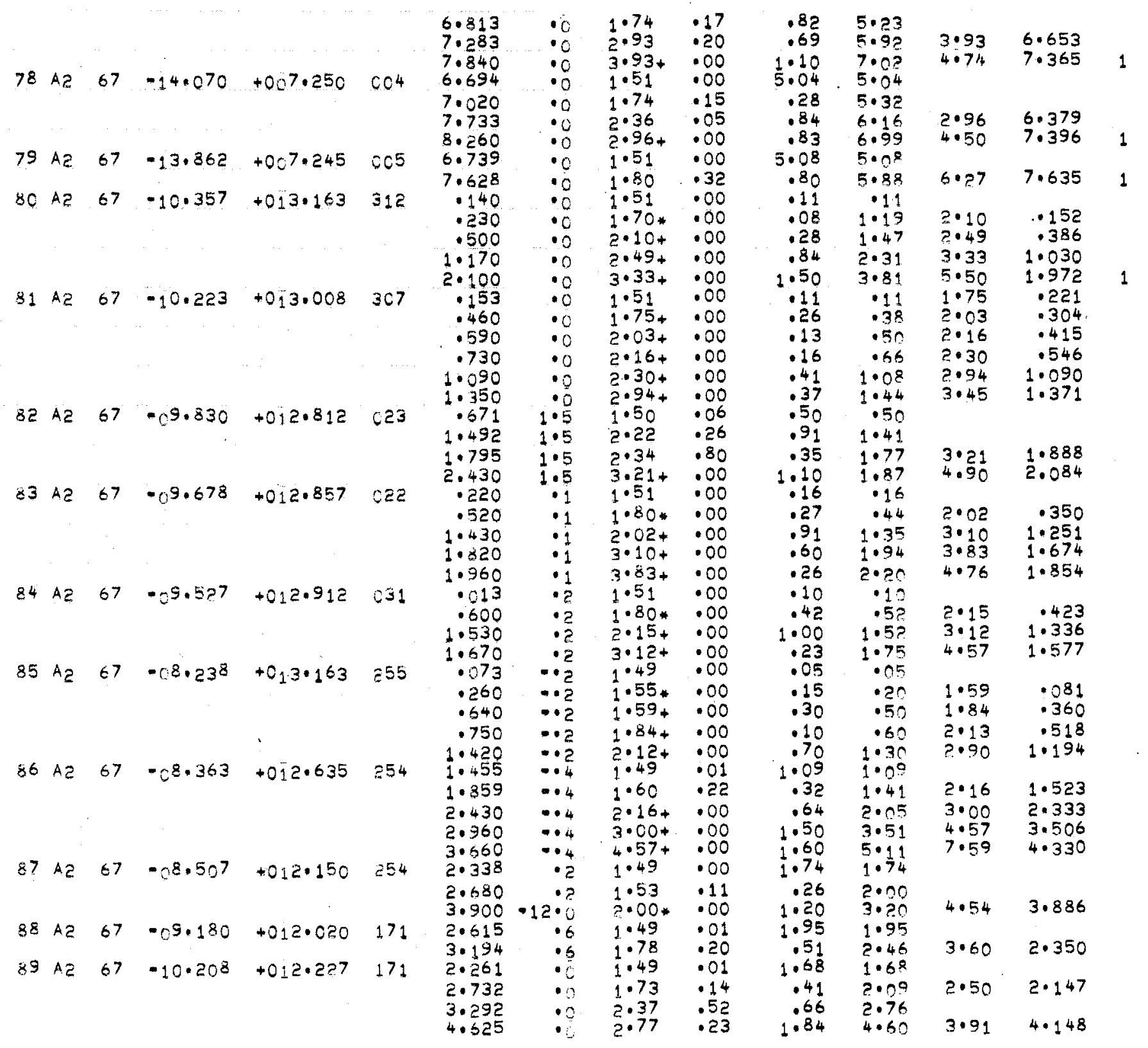

REFRAC REFRAC BASE VELAC INTERC MENT KMISEC SEC IN TIME OIP VELEC VELOC DES KM/SEC KM/SEC

HICK THICK 
$-25-$

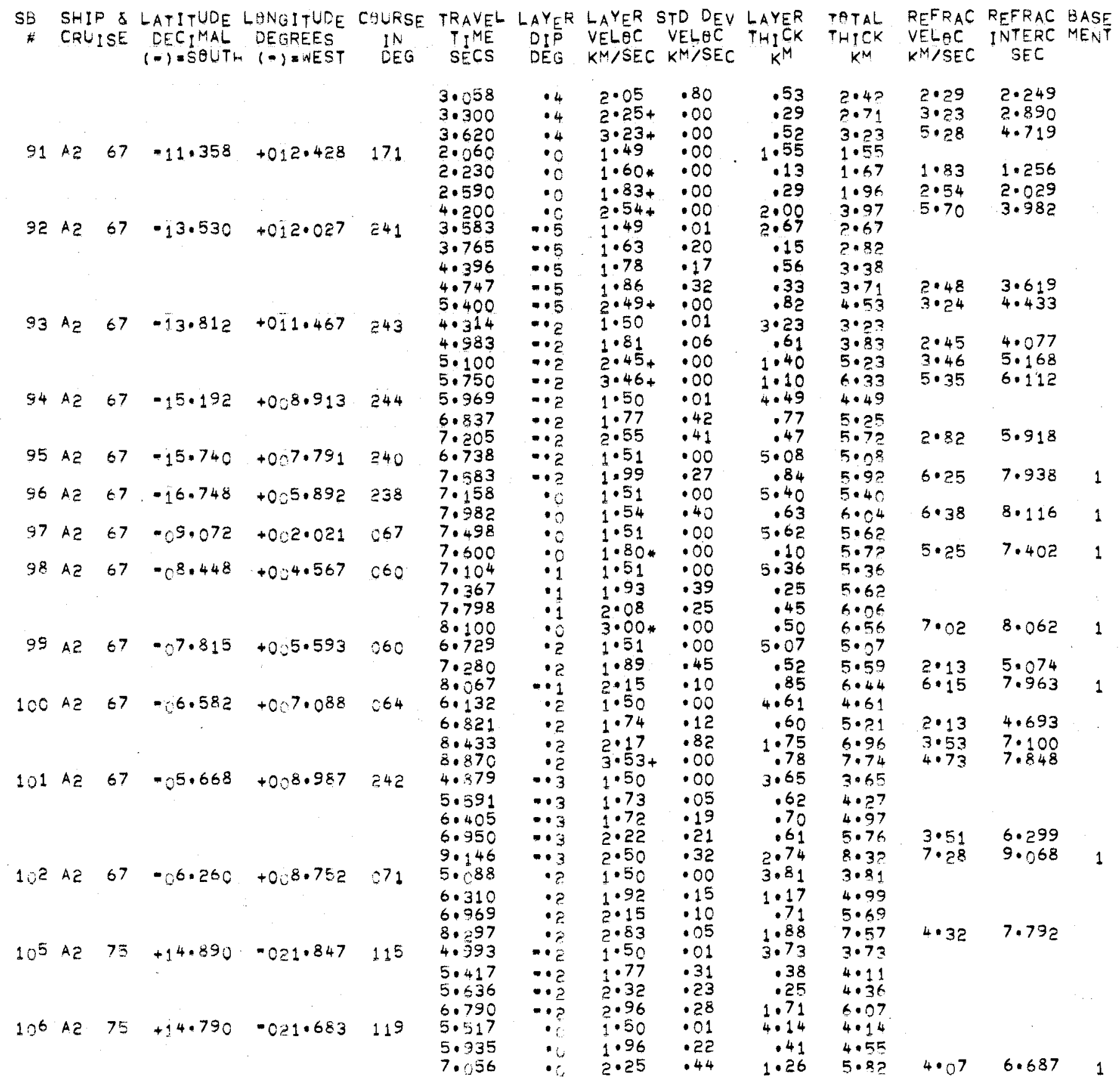


SB SHIP S LATITUDE LONGITUDE CQLRSF TRAVEL LAYER LAYER STD DEV LAYER$$
\begin{array}{r}
\text { * CRUISE DECIMAL DEGREES IN } \\
(-)=S Q U T H \quad(-)=\text { INEST DEG }
\end{array}
$$

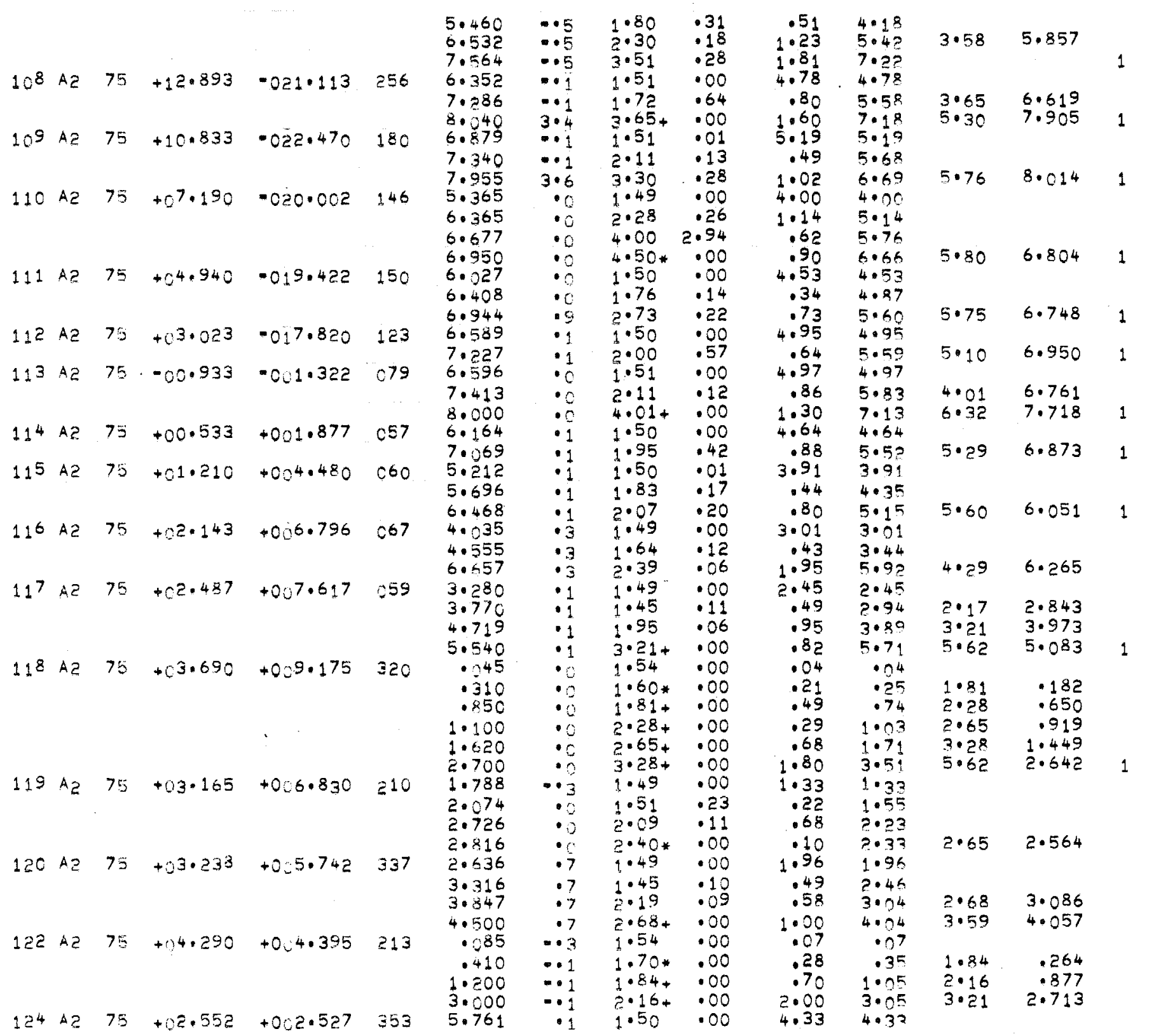

TETAL.

TIME OIP VELOC VELOC THICK THICK SECS

DIP
KM KM

REFRAC REFRAC BASE VELAC INTERE MENT KMISEC SEC 


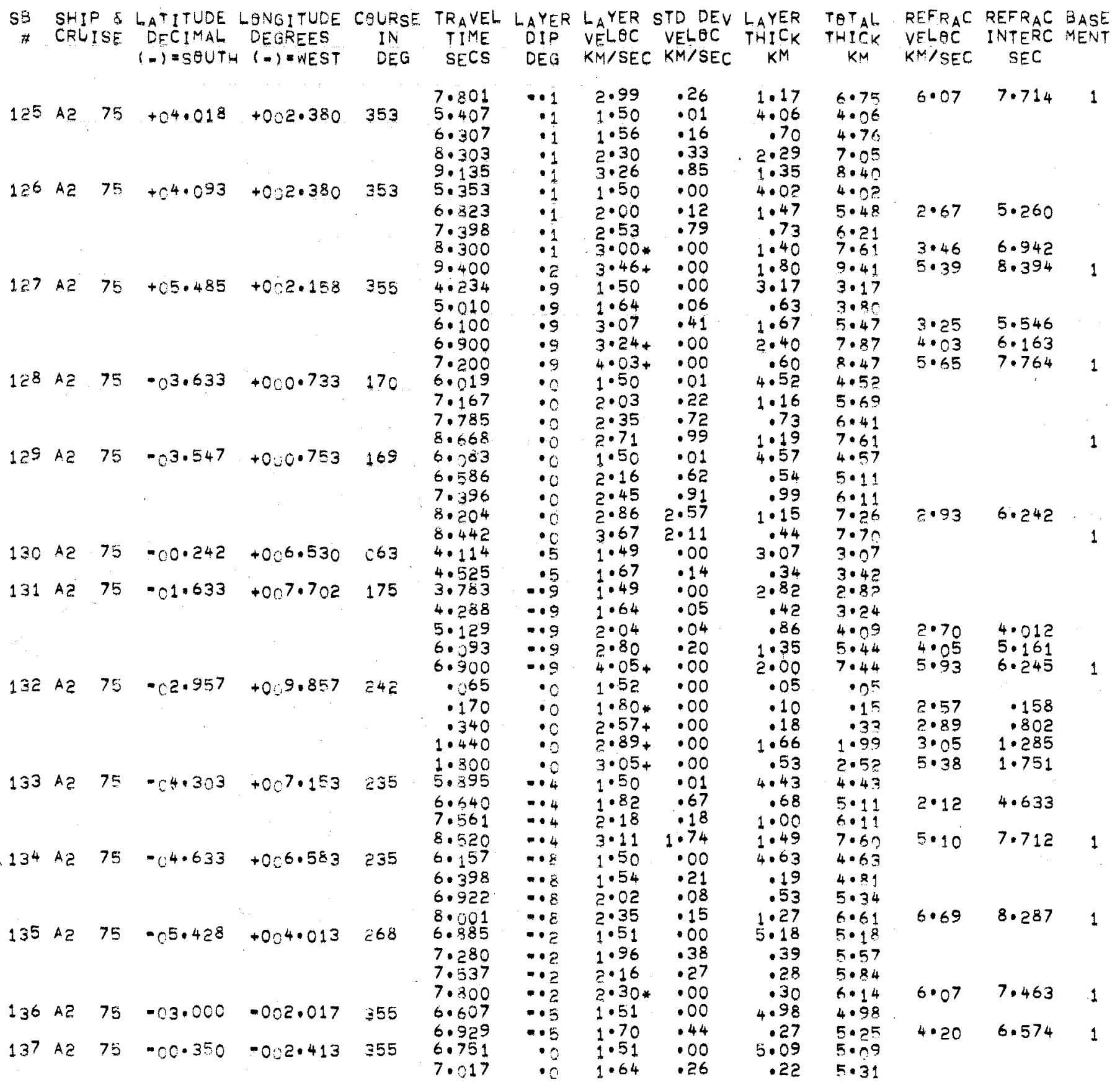


SE SHIF S LATITUDE LONGITUDE COLRSE TRAVEL LAYER LAYER STD DEV LAYER TOTAL RER

* CRUISE DECIMAL DEGREES IN TIME DIP VELGC VELOC THICK THICK $(-)=S Q U T H \quad(-)=$ WEST DEG SECS

138 A2 $75+02.155 \quad-022.527 \quad 000$

139 A2 $75 \quad+03.840 \quad-005.343 \quad 183$

141 A2 $75+06.230 \quad \cdot 010 \cdot 892 \quad 208$

42 AE $75+05.135 \quad-011 \cdot 375 \quad 212$

43 A2 $75+04.833 \cdot 011.547 \quad 212$

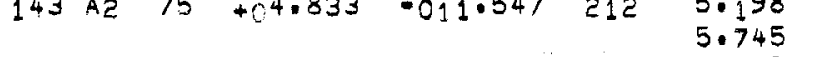

$6 \cdot 239$

7.130

$7 \cdot 240$

7.430

$144 \mathrm{~A} 275+3.812 \quad \cdot 012 \cdot 105 \quad 212$

5.883

6.627

7.973

145 A2 $75+22,460 \quad-012.858 \quad 210$

6.538

7.502

$7 \cdot 325$

7.744

8.205

$146 \mathrm{A2} 75+03.547 \quad 0.0140488 \quad 312$

6.310

7.720

7.290

7.600
6.568

$147 \mathrm{A2} .7 \mathrm{~b}+4.363 \quad-015 \cdot 088 \quad 030$

$7 \cdot 345$

7.554

7.004

$148 A_{2} 75+5.833 \quad-014.237 \quad 032 \quad 6.270$

6.763

7.406

7.933

$149 \mathrm{~A} 275+6.840 \quad-013.547 \quad 039$

$5 \cdot 260$

5.919

6.248
7.620
DEC

:0

$-1 \cdot 0$
$-1 \cdot 0$

$-1 \cdot 0$

$-1 \cdot 0$

$-1 \cdot 0$

$-1 \cdot 0$

10

?

$2.00 * .00$

$3.49+.00$

- $1.54 \quad .00$

. 0 1.80* .00

- $2.34+.00$

. $3.51+.00$

. .5

. .5

1.64

$\begin{array}{ll}1.50 & .00 \\ 1.64 & .09\end{array}$

$\begin{array}{ll}1.64 & .09 \\ 2.20 & .16\end{array}$

.140 .17

$.43 .76+.00$

$\begin{array}{lll}. .3 & 3.50+.00\end{array}$

$\begin{array}{lll}. .3 & 1.52 \quad .07\end{array}$

. 32.03

.$-3 \quad 2.72$

4.05

$.34 .83+.00$

1.50

- -1

. $1 \quad 1.50$

.1 3.05

.21 .51

- 2.08

$\because 2.11$

- 2.67

.0
.0

0.1

$\because 1$

$-1$

$\because$

.0

$3 \cdot 5$

$3 \cdot 6$

.

$:$

$: 1$

$1 \cdot 2$

$1 \cdot 2$

$1 \cdot ?$

1.51 .01
- 0 2.79+.00
1.60

2. 28

$5.50+.00$

1.51 .00

$2 \cdot 31 \cdot 1.05$

2.681 .64

$5.23+.00$

$1.70 \quad .33$

$2.40 \quad .19$

$3 \cdot 06 \quad .31$

$1.50 \quad .00$

$1 \cdot 80$

$2 \cdot 21$

3.00*
5.09

$1.13 \quad 6.46$

$.91 \quad 7.37$

3.82 3182

.594 .41

1.25

.71

1.60

.07

.06

.87

.07

.07

.33

.66

.70

3.64

.57

.45

$1 \cdot 17$

$3 \cdot 10$
3.90

3.90
.42

.50

$1 \cdot 21$

.60

1.40

4.42
.72

.72
2.05

4.92

.48

.34

.56

.88
4.76

4.76
.56

.30

.77
4.95

.90

.41
.43
4.72

.42

.77

.81
3.95

.59

.36

1.50

7.19

$4 \cdot 76$

5.32

$4 \cdot 95$

$5 \cdot 85$

$5 \cdot 14$

$5 \cdot 91$

$3 \cdot 95$

$4 \cdot 54$
REFRAC REFRAC BASE

VELGC INTERE MENT

$K M / S E C$ SEC

$\begin{array}{lrr}2.66 & 6.392 & \\ & & 1 \\ 2.21 & 4.655 & \\ 3.96 & 6.567 \\ 4.22 & 6.969 & \\ 5.31 & 7.911 & 1 \\ 3.49 & .143 \\ 4.87 & 1.643 \\ 2.34 & .113 \\ 2.79 & .359 \\ 3.51 & .816 \\ 4.27 & 1.218\end{array}$

$3 \cdot 76 \quad 5.894$

$5.60 \quad 7.780 \quad 1$

$3 \cdot 18 \quad 5 \cdot 570$

$4.02 \quad 6.513$

$4.83 \quad 6.956$

$6.47 \quad 7.711 \quad 1$

$2.93 \quad 6.038$

$5 \cdot 33 \quad 7.742 \quad 1$

1

$5.5 ? \quad 5.50 \quad 7.100$

$\begin{array}{llll}6.39 & 6.46 \quad 7.380 & 1\end{array}$

$6 \cdot 2^{5} \quad 5 \cdot 23 \quad 7 \cdot 352$

$6.69 \quad 6.29 \quad 7.628 \quad 1$

$6.72 \quad 5.68 \quad 7.766 \quad 1$

$6.40 \quad 5.30 \quad 7.401 \quad 1$ 
SB SHIP \& LATITUDE LONGITUDE COLASE TRA * CRUISE DECIMAL DEgREES $(-)$ sSOUTH $(-1)$ WEST
IN DEG $\begin{array}{llllll}151 & A 2 \cdot 75 & +06.962 & -014 \cdot 107 \quad 248\end{array}$

$152 A_{2} 73+06.520=015 \cdot 008 \cdot 249$

$153 A_{2} 75+05.647 \quad-016.590 \quad 245$

$\begin{array}{llllll}154 \mathrm{AL} \quad 75 & +04.838 & .018 .300 \quad 243\end{array}$

$155 \mathrm{AZ} 75+04.268 \quad-019.415 \quad 243$

$\begin{array}{lllll}156 \text { A2 } & 75 & +03.903 & -019.980 & 311\end{array}$

$157 \mathrm{AE} \quad 75+04.367-020.577 \quad 312$

$158 \mathrm{Az} 75+04.820 \quad-021 \cdot 088 \quad 314$

$\begin{array}{llll}159 \mathrm{AZ} & 75+05.475 & -021.778 \quad 312\end{array}$

160 A. $75+06.663-023.170 \quad 058$

$\begin{array}{lllll}161 A_{2} & 75+07.445 & -021 \cdot 928 & 068\end{array}$

$162 A 275+08.300 \quad-020.247 \quad 060$

163 AE $75+08.535-019.758 \quad 055$

$164 \mathrm{Az} \quad 75+9.133 \quad-018.860 \quad 55 \quad 6.199$
TRAVEL LAYER LAYER STD DEV LAYER

TIME SECS

.970

5.526

6.490

7.810

6.743

$7 \cdot 375$

8.000

7.184
7.318

7.318
6.618

7.385

$6 \cdot 018$

6.668

7.223
6.287

6.087
6.453

6.882

7.612

7.892

$5 \cdot 041$

5.719

6.229
3.698

$4 \cdot 144$

4.638

5. 223

$5 \cdot 394$

5.630

6.190
4.848

$5 \cdot 385$

5.750

5.975
4.558

5.111

5.503

5.973
6.773

6.773
5.678

5.378

6. 201

6.980

5. $\$ 63$

$6 \cdot 124$

DIP VELOC VELOC THICK

- $1.80 * .00$

$\begin{array}{rrr}.0 & 1.80 * & .00 \\ .0 & 2.29+ & .00 \\ .5 & 1.50 & .01\end{array}$

. $.5 \cdot 1.76 \quad .11$

$.05 \quad 2.14 \quad .27$

$.53 .50 * .00$

. $11.51 \quad .00$

$.11 .68 \quad .04$

$0.1 .87 \quad .17$

$\because 1 \quad 2.74+.00$

$.0 \quad 1.85 \quad .05$

$2 \cdot 6 \quad 2 \cdot 57 \quad \cdot 32$

. 1.51 .00

$.0 \quad 2.01 \quad .25$

$\begin{array}{lll}.0 & 3.86 & 1.30\end{array}$

$1 \cdot 9$

$-3 \cdot 2$

$-6 \cdot 7$

-

$:$

$.123 .16 \quad .16$

$1.7 \quad 1.50+.01$

$1.7 \quad 1.99 \quad .12$

$1.7 \quad 4.23+.00$

$.8 \quad 1.49 \quad .00$

- $1.80 \quad .41$

$\begin{array}{rr}.8 & 2.58 \\ .19 & .71\end{array}$

$.0 \quad 1.50 \quad 15$

$.91 .98 \quad .20$

$. .92 .30+.00$

$. .94 .10+.00$

. $1.50 \quad .00$

$\begin{array}{lll}.8 & 1.71 & .36 \\ .8 & 2.49 & .16\end{array}$

$.8 \quad 2.80 \quad .61$

$\begin{array}{lll}-0 & 1.50 & .00 \\ .0 & 2.02 & 13\end{array}$

$.0 \quad 2.96 \quad .17$

$.03 .48+.00$

$.04 .01+.00$

- $01.50 \quad .01$

- 1.82 .12

- $2.70 \quad .29$

$.03 .80 * \quad .00$

$\begin{array}{lll}\because 1 & 1.50 & .00 \\ .1 & 1.67 & .11\end{array}$

$\because 1 \quad 3.15 \quad .40$
.01

$4 \cdot 15$

.85

.61

1.40

4.79

.32

.59

1.95

$4 \cdot 97$

.54

.81

4.99

.77

$1 \cdot 22$

4.71

.38

.57

4.58

.32

.46

1.15

3.78

3.78
.68

1.08

2.76

.40

.44

3.47

.50

.17

.27

1.20
$3 \cdot 63$

.46
.45

.45

.56

.73

.64

1.60

$4 \cdot 27$

.27

.30

.41
4.41

4.41
.22

1.53
4.67
3.41
TQTAL

THICK KM

REFRAC REFRAC BASE

VELAC INTERC MENT KMISEC SEC

$\begin{array}{llll}.06 & 2.29 & .059 \\ .22 & 2.79 & .170 & \\ 4.15 & & & \\ 5.00 & & & \\ 5.60 & & & \\ 7.01 & 6.22 & 7.677 & 1 \\ 4.79 & & & \\ 5.11 & & & \\ 5.70 & 2.74 & 6.245 & \\ 7.65 & 4.25 & 8.317 & \\ 4.97 & & & \\ 5.52 & & & \\ 6.33 & 6.44 & 7.853 & 1 \\ 4.99 & & & \\ 5.76 & & & \\ 6.98 & 7.18 & 8.072 & 1\end{array}$

$4 \cdot 71$

$5 \cdot 09$

$5 \cdot 65$

4.58

$4 \cdot 90$

$5 \cdot 36$

$6 \cdot 52$

$7 \cdot 23$

$4 \cdot 45$
5.53

2.76

$3 \cdot 16$

$3 \cdot 61$
$3 \cdot 47$

$3 \cdot 98$

$4 \cdot 15$

$4 \cdot 4$ ?

$5 \cdot 62$

$3 \cdot 63$

4.09

$4 \cdot 55$

$4 \cdot 8 \mathrm{~A}$

$3 \cdot 41$

$3 \cdot 97$

4.70

$5 \cdot 34$

$6 \cdot 94$

$4 \cdot 27$

$4 \cdot 54$

4.84

$5 \cdot 25$

$4 \cdot 41$

4.63

$6 \cdot 15$
$4 \cdot 67$

$7.25 \quad 7.572$

$4 \cdot 24 \quad 5.435$

$5 \cdot 50 \quad 6.085$

$2 \cdot 25 \quad 3 \cdot 242$

$2.90 \quad 3.585$

$2 \cdot 30 \quad 4 \cdot 102$

$4 \cdot 10 \quad 5.414$

$5 \cdot 70 \quad 6.116 \quad 1$

$5 \cdot 16 \quad 6.200$

$2.96 \quad 4.560$

$3.48 \quad 4.880$

4.015 .391

$5 \cdot 94 \quad 6.389$

1

PAGE 11

EASTERN ATLANTIC CONTINENTAL MARGIN PRGGRAM

R/V ATLANTIS II 67 AND 75 
SB SIIF $\&$ LATITUDE LGNGITUDE CALRSE TRAVEL LAYER LAYER STD DEV LAYER CRUISE DECIMAL DEGREES IN TIME
$(-)=S O U T H$
$(N)$ NEST DEG SECS OIP VELOC VELOC THICK DES KM/SEC KM/SEC KM

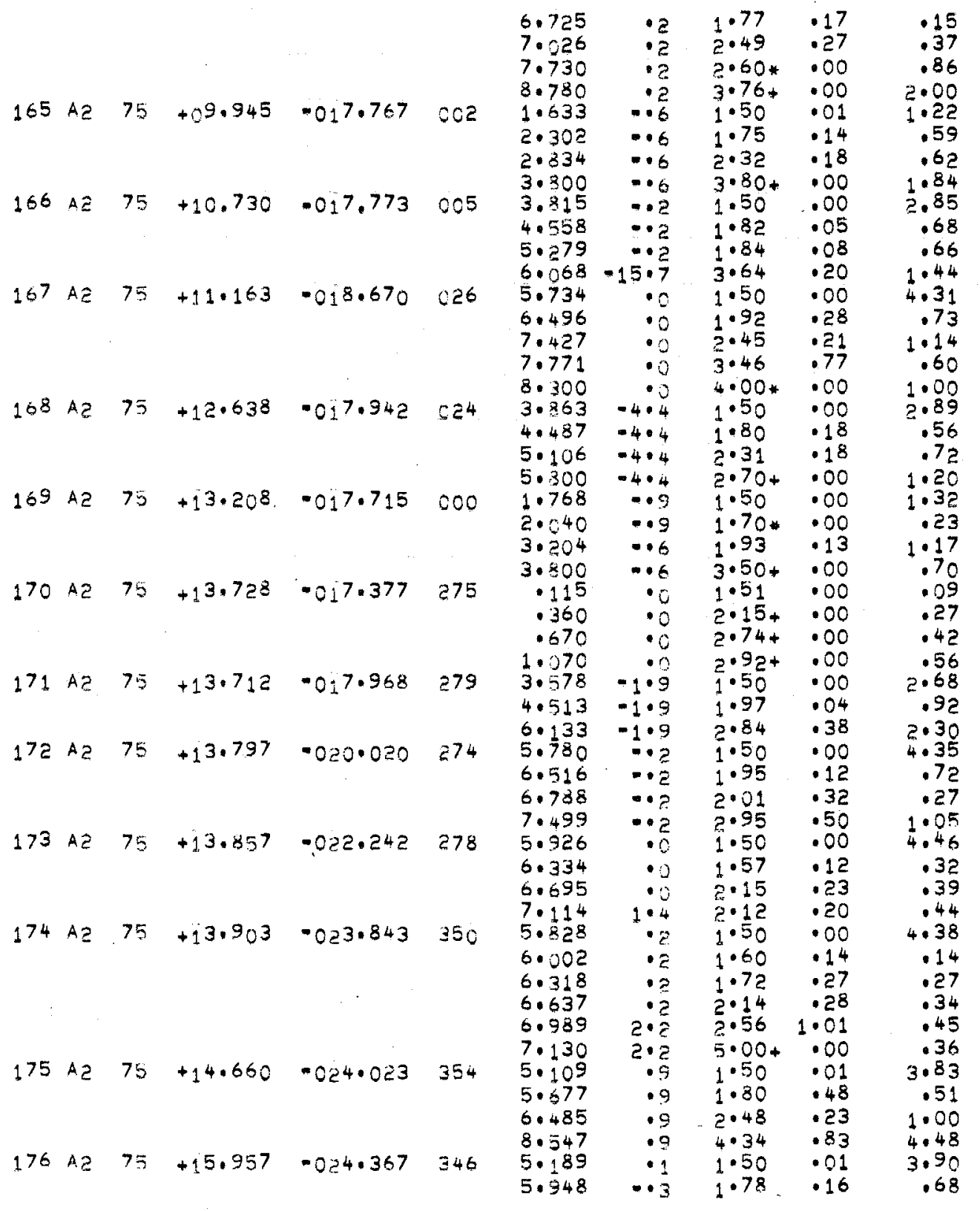

PAGE 12
TOTAL THICK. $K M$

REFRAC REFRAC BASE VELOC INTERC MENT $\mathrm{KM} / \mathrm{SEC}$ SEC

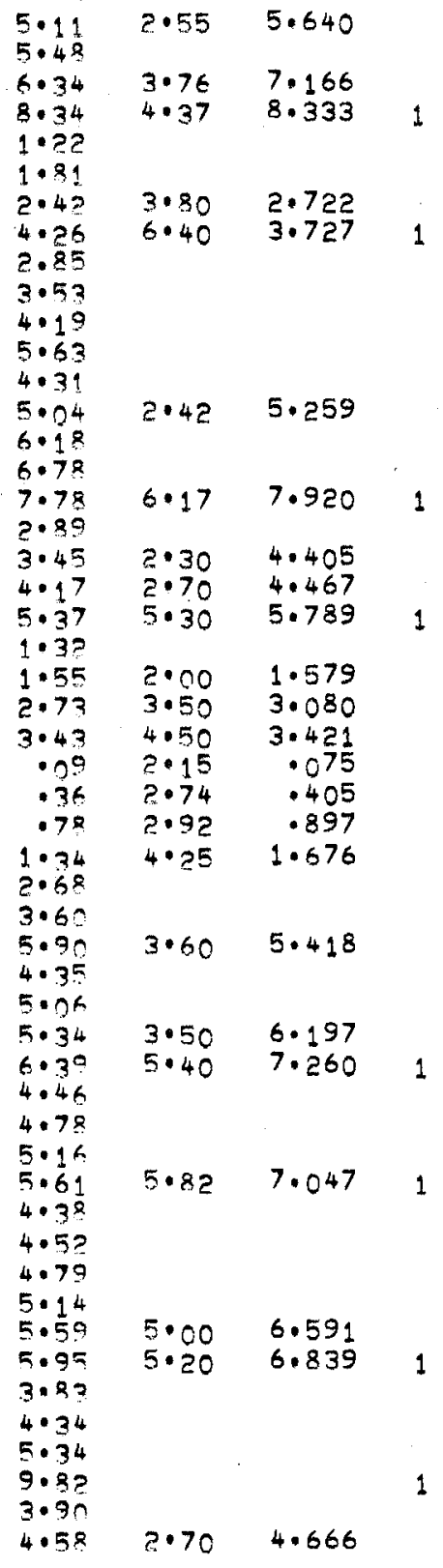

R/V ATLANTIS II 67 AND 75 
SB SHIP \& LATITUDE LONGITUDE COURSE TRAVEL LAYER LAYER STD DEV LAYER \# CRUISE DECIMAL DEGREES IN TIME $(-)=S Q U T H(-)=N E S T$ CEG SECS

177 A2 $75+\$ 7.858-024 \cdot 753 \quad 100$

178 A2 $75+17.613=023.782 \quad 104$

179 A2 $75+16.707-020.528 \quad 105$

150 A2 $75+16.327 \quad .019 .187 \quad 100$

$81 \mathrm{A2} \quad 75+15.897 \quad-0 \overline{7} .500 \quad 103$

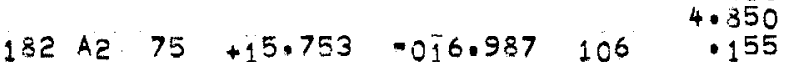

$7 \cdot 100$

5.649

5.800

4.748

$5 \cdot 060$

5.800

4.743

$5 \cdot 431$

6.219

6.364

6.314

$7 \cdot 200$

$4 \cdot 622$

$5 \cdot 177$
$5 \cdot 748$

6.527

7.168

7.600

3.278

3.675

4.411

.260

.510

.790

.940

$1 \cdot 850$

183 A2 $75+17.115-016.643 \quad 645$

- 135

.400

$1 \cdot \geq 10$

2.710

$184 \mathrm{A2} \quad 75+17.537 \quad 016 \cdot 525 \quad 318 \quad .188$

1.180

1.320

1.750

2.290

185 A2 $75+17.932=016.990 \quad 281$

5.285

3.792

186 A2 $75+18.108=018.170 \quad 288$

3.358

4.617

4.940

$5 \cdot 330$

5.750

187 A2 $75+19.430 \quad-023.992 \quad 275$

5.535

5.845

0.326

$\begin{array}{lllll}188 \text { A2 } 75+21.105 & -024.442 \quad 110 \quad 6.473\end{array}$

FAGE 13
DIF VELOC VELOC THICK THICK DEG

$\begin{array}{rrrr}-3.2 & 3.20 * & .00 & .39 \\ .3 & 1.50 & .00 & 3.69 \\ .8 & 1.43 & .22 & .53\end{array}$

$\begin{array}{lll}.8 & 1 \cdot 43 & .22 \\ .8 & 3 \cdot 30+ & .00\end{array}$

$1.1 .50 \quad .01$

$1.1 .59 \quad .41$

$.1 \quad 1.85 . .22$

.1 .1 .50 .00

$.1 .1 .14 \quad .17$

.12 .31 .11

$.1 \quad 2.88 \quad .00$

$.1 \quad 3.51 \quad .19$

.1 4.20* .00

- 2 1.50 .00

$.2 \quad 1.48 \quad .07$

$\begin{array}{lll}.2 & 1.55 & .41 \\ .2 & 2.26 & .25\end{array}$

. $3.47 \quad 4.12$

$.24 .67+.00$

$1.3 \quad 1.50 \quad .00$

$1.3 \quad 1.78 \quad .09$

$\begin{array}{lll}1.3 & 2.05 & .15 \\ 1.3 & 2.85+.00\end{array}$

$1.3 \quad 2.85+.00$

. 2 1.80. .00

. $1.97+.00$

. $2.41+.00$

. $3.18+.00$

$.23 .47+.00$

. $1.50 \quad .00$

. $1.80 * .00$

.0 $2.04 \quad .00$

$.02 .84 \quad .00$

$.3 \quad 1.51 \quad .00$

. $.3 \quad 1.70 * .00$

$. .3 \quad 1.82+.00$

$.02 .01+.00$

$.032 .48+.00$

$-1 \cdot 3$

$-1 \cdot 3$

$-1 \cdot 3$

1.50

1.46

.00

$1.46 \quad .06$

$1.50 \quad .00$

1.89

2.06

.00
.08

.08

2.21 .50

$2.89+.00$

$1.51+.00$

1.63

.18

1.92 .10

$2.61: 13$

1.51
6.01

3.69

$4 \cdot 21$

$4 \cdot 46$

3.57

.25

.50

.41
3.56

.74

.91

.21

.79
.77

3.47

.41

.44
.88

.88

1.11

2.45

.35

.75

.62

.09

.25

.25

1.60

1.60
.10

.24

.81

$2 \cdot 10$

.14
.87

.87

.13
.43

.68

1.71
.29

.93
2.89

2.89

.72

.33
.43

.61
4.17

$4 \cdot 17$

.25
.46

.60
4.90

$3 \cdot 47$

3.89

$4 \cdot 32$

$5 \cdot 20$

$6 \cdot 31$

$7 \cdot 31$

2.45
2.81

$3 \cdot 56$

$4 \cdot 18$

.12

-21
.46

.46

1.05

$2 \cdot 65$

.10

$1 \cdot 15$

$3 \cdot 25$

1. 14

1.01

1.14
1.57

$2 \cdot 25$

$1 \cdot 71$

2.00,

2.93
2.89

$2 \cdot 89$

3.61

3.94

$4 \cdot 37$
4.98

4.98

4.42

$4 \cdot 48$

5.49

5.49
4.97
TETAL

REFRAC REFRAC BASE

VELAC INTERC MENT

$\mathrm{KM} / \mathrm{SEC}$ SEC

$\begin{array}{lll}4.90 & 6.029 & 1 \\ 3.30 & 5.466 & \\ 6.20 & 5.464 & 1 \\ & & \\ 4.31 & 5.388 & \\ 4.70 & 5.618 & 1\end{array}$

$5.64 \quad 6.493 \quad 1$
$2.41 \quad 4.359$

$3 \cdot 14 \quad 5.293$

$4.67 \quad 6.209$

$6.096 .952 \quad 1$

$2 \cdot 85 \quad 3.442$

$4 \cdot 30 \quad 4 \cdot 308$

$1.97 \quad 168$

$\begin{array}{ll}1.41 & .610\end{array}$

$3.47 \quad .851$

6.251 .800

$2.04 \quad .200$

$2.84 \quad .875$

4.11 2.286

$1.82 \quad .075$

$2.01 \quad .180$

$2.48 \quad .559$

?. $98 \quad 1.082$

$2 \cdot 30 \quad 2 \cdot 476$

$2 \cdot 17 \quad 3 \cdot 263$

$2.89 \quad 4.605$

$3.99 \quad 5.417$

$5.51 \quad 6.857 \quad 1$

R/V ATLANTIS II 67 AND. 75 


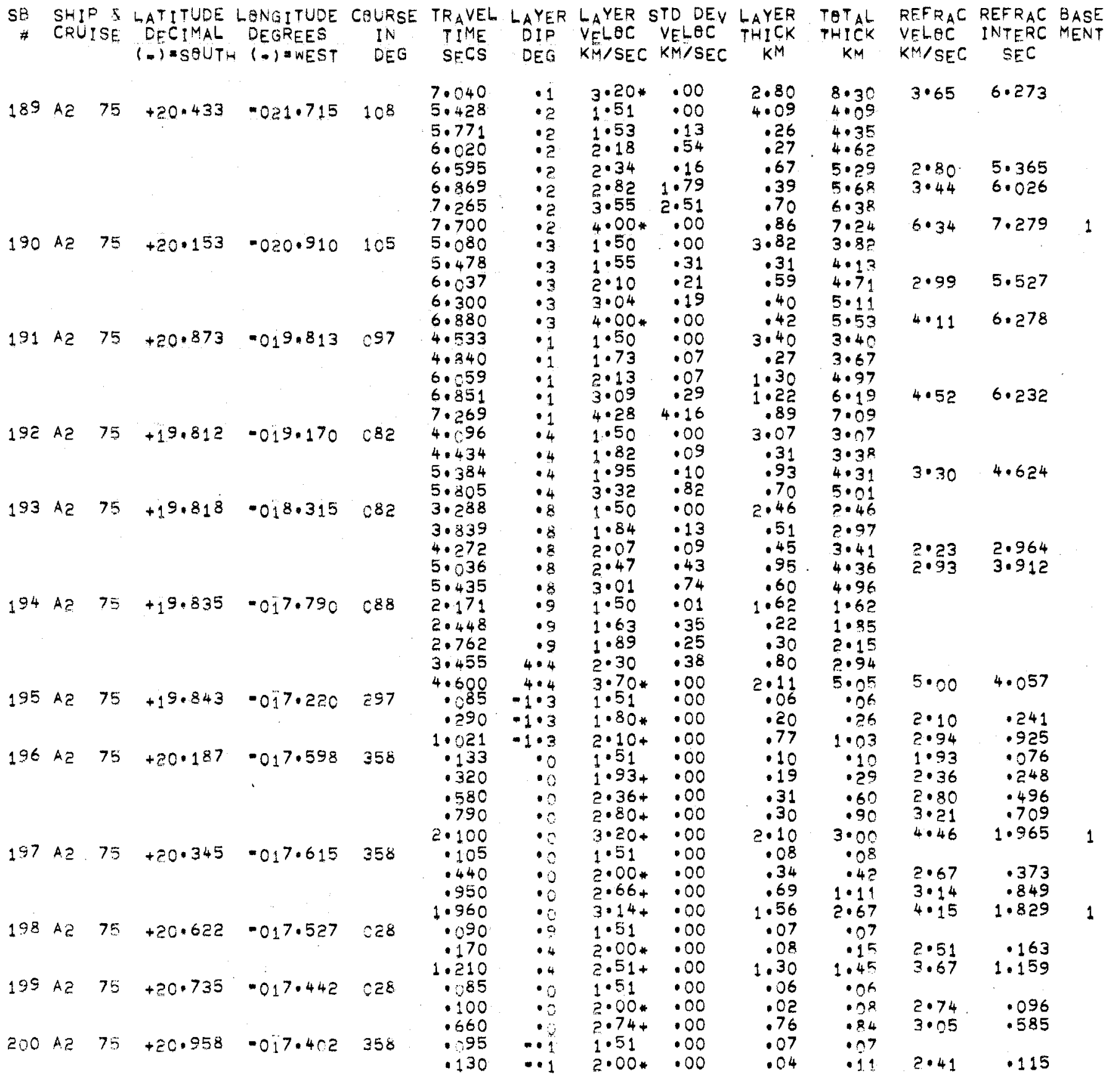


SE SHIP \& LATITUDE LONGITUDE COLRSE TRAVEL LAYER LAYER STD DEV LAYER

* CRUISE DETTME LONGITUDE COURSE TRAVEL LAYER LAYER STO DEV LAYER $(-)=$ SQUTH $(-)=$ WEST DEG SECS DEG

$K M / S E C K M / S E C \quad K M$$$
\begin{aligned}
& 0 \\
& 1.56 \\
& 4 . \\
& 5 . \\
& 6 . \\
& 5
\end{aligned}
$$$$
203 \text { A2 } 75+21.616=020.703 \quad 28
$$

TATAL

THICK $K M$

.11

.11

.34

$1 \cdot 12$

$3 \cdot 34$

.49

$1 \cdot 0.3$

1.10
.25

4.21

.40

.59

. .85

1.84
4.52

.56
.64

.64
.75

.75
4.95

.38

1.06

.30

$2 \cdot 40$

.37

.13

1.08

1.02

3.51

.83

1. 52

.76

.03

.06

. 31

1.24

1.43

$2 \cdot 33$

.86
1.10

1.08
.38

.38
4.11

1.83

1.45

1.06

.63

.40
1.25
.08

.15

.03

.09

.40

$1 \cdot 54$

$3 \cdot 07$

$3 \cdot 2$ ก

$4 \cdot 29$

$5 \cdot 37$
$5 \cdot 76$

$5 \cdot 76$

$4 \cdot 11$

$5 \cdot 95$
$7 \cdot 30$

$1 \cdot 06$

1.68

$2 \cdot 0 R$
$3 \cdot 33$

$3 \cdot 33$
REFRAC REFRAC BASE VELAC INTERC MENT $\mathrm{KM} / \mathrm{SEC}$ SEC

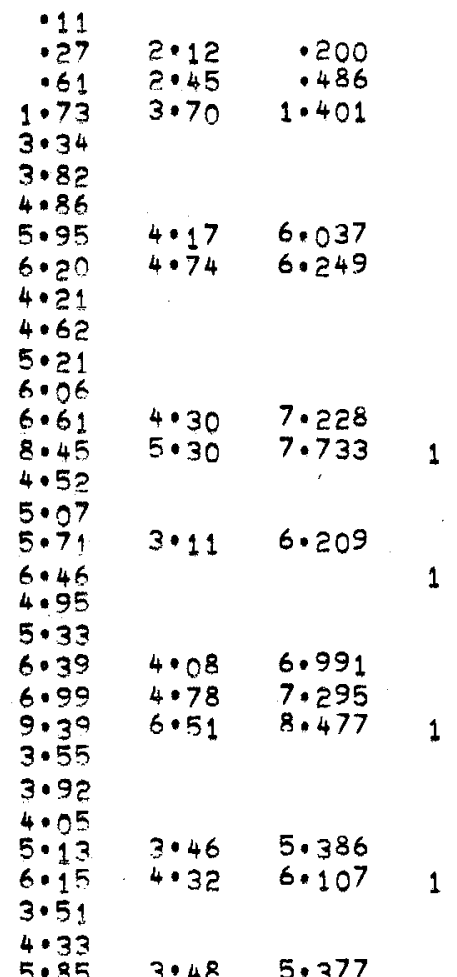

$5.35 \quad 3.48 \quad 5.377$

$6.61 \quad 4.59 \quad 6.197$

.23

$2 \cdot 30 \quad .098$

$2.81 \quad .337$

$4 \cdot 39 \quad 1.202$

$5 \cdot 18 \quad 1.846 \quad 1$

$3.50 \quad 4.524$

$3.80 \quad 4.814$

$2.80 \quad 3.218$

$5.70 \quad 5.380$

1.931 .030

$5 \cdot 10 \quad 3.291 \quad 1$

2.44 .201

FAGE 15

EASTERN ATLANTIC CONTINENTAL MARGIN PREGRAM

R/V ATLANTIS II 67 AND 75 

SB SHIP \& LATITUDE LONGITUDE CQURSE TRAVEL LAYER LAYER STD DEV LAYER
\# CRUISE DECIMAL OEGREES IN TIME DIP VELGC VELOC THICK $(-)$ SSOUTH (-) IEEST DEG SECS DEG KM/SEC KM/SEC

$213 A 275+22.917-017.093 \quad 293$

1.390

1.860

$21^{4} A E \quad 75+23.017 \quad 017.877 \quad 298$

3.897

$5 \cdot 026$

5.900

$215 \mathrm{~A} 275+23.420=018.460 \quad 297$

6.200

3.899

4.304

$4 \cdot 540$

6. 148

$\begin{array}{lllll}216 & A_{2} & 75 & +23.667 & -019.195 \quad 295\end{array}$

$4 \cdot 508$

4.952

5.456

6.080

$217 A_{2} 75+23.948 \quad-019.930 \quad 294$

6.710

4.983

5.809

6.539

$7 ., 300$

$21^{8}$ A2 $75+24.528 \quad-021.632 \quad 292$

219 A2 $75+24.863-022.587 \quad 293$

6.419

6.674

7.226

7.620

$6 \cdot 433$

$7 \cdot 042$

7.409

$8 \cdot 139$

220 A2 $75+25.067 \quad 023 \cdot 128 \quad 292$

7.487

7.941

221 A. $75+25.707-025 \cdot 037 \quad 296$

6.355

$22^{3} A 2^{25}+0^{8.048}=029.558 \quad 2^{9}$

7.080

6.533

6.738

$6 . \times 70$

6.373

6.342

6.950

225 A2 $75+30 \cdot 330 \quad-027 \cdot 220 \quad 070$

226 Az $75+30.715 \quad-025.992 \quad 072$
.138

- 210

- 900

1.640

$3 \cdot 118$

6.083

7.173

$\begin{array}{rll}10 & 3.04+ & .00 \\ 10 & 4.60+ & .00 \\ 10 & 1.51 & .00 \\ 10 & 1.80 * & .00 \\ 10 & 2.88+ & .00 \\ 10 & 3.30+ & .00 \\ .7 & 1.50 & .00 \\ \therefore 7 & 1.92 & .07 \\ 17 & 2.32 & .15 \\ 1.7 & 3.33 & .49 \\ 1.7 & 3.60 * & .00 \\ 1.7 & 4.30+ & .00 \\ 1.4 & 1.50 & .00 \\ 1.4 & 1.72 & .19 \\ 1.4 & 1.96 & .10 \\ 1.4 & 2.50 & .22 \\ 1.4 & 2.86 & .51\end{array}$

. $.6 \quad 1.51 \quad .00$

. $.6 \quad 1.73 \quad .16$

$.06 \quad 2.02 \quad .61$

$.06 \quad 2.12 \quad .15$

$\begin{array}{lll}-.6 & 3.77 \quad 1.31\end{array}$

$\begin{array}{lll}0.06 & 1.51 & 00\end{array}$

$\because 2 \quad 1.67 \quad 116$

$\begin{array}{rrr}\because 2 & 2.17 & .30 \\ \cdots 2 & 3.39 & 1.93\end{array}$

$\begin{array}{rl}\because 2 & 3.39 \\ \because .93 & 4.50+\end{array}$

a. $1.51 \quad .01$

$.0 \quad 1.68 \quad .13$

$\begin{array}{rrr}-1.2 & 1.63 \\ .1 .8 & .28\end{array}$

- $1.8 \quad 4.20+.00$

$\because 1 \quad 1.51 \quad .00$

$\begin{array}{lll}0.1 & 1.79 \quad .07\end{array}$

$.012 .18 \quad .16$

$\begin{array}{lll}-9.6 & 3.60 \quad .87\end{array}$

- $1.51 \quad .00$

- $1.83 \quad .08$

$\begin{array}{rrr}.0 & 1.95 & .52 \\ 1.6 & 2.69 & .59\end{array}$

$\begin{array}{lll}1.6 & 2.69 & .59 \\ .0 & 1.51 & .00\end{array}$

$2.5 \quad 1.80 * .00$

$-1.4 \quad 1.51 \quad: 01$

$-1.4 \quad 1.84 \quad 1.28$

$\begin{array}{lll}-1.4 & 3.00+.00\end{array}$

. $1.97 \quad .26$

$.0 .71+.00$

$\begin{array}{ll}7.71+ & .00 \\ 1.51 & .00\end{array}$

$\begin{array}{llll}6.570 & -11.9 & 2.80 * & .00 \\ 7.271 & .0 & 1.52 & .00 \\ 7.601 & -4.0 & 1.86 & .97\end{array}$

$\begin{array}{llll}6.570 & -11.9 & 2.80 * & .00 \\ 7.271 & .0 & 1.52 & .00 \\ 7.601 & =4.0 & 1.86 & .97\end{array}$
... $1.63 \quad .23$

. $1.51 \quad .01$
TOTAL

THICK KM

1.04
1.07

.10

.06

1.01

1.22

2.34

.75

1.03

.40

1.57

.65

2.93

.35

.23

$1 \cdot 43$
$3 \cdot 39$

$3 \cdot 39$

.51

.66

$1 \cdot 19$

3.76

.69
.79

.72

4.60

.28

-21

.55
.83

.83
4.86

4.86
.54

.40

1.31

5.02

.49

.31

.61
5.19

.20
4.94

. 19

.22
4.32

.46

.21

4.80
.31

5.51

. 31

1.17

$1 \cdot 39$

2.34

$4 \cdot 1$ ?

$4 \cdot 5$ ?

6.09

6.74

2.93

3.28

$3 \cdot 51$

$4 \cdot 27$

5.70
3.39

$3 \cdot 78$

5.96

6.72

$4 \cdot 60$

5.09

$5 \cdot 64$

6.61

4.86

5.80

7.12

$5.0 ?$

5.51

5.30

$5 \cdot 13$

4.82

$5 \cdot 28$

5.79

$5 \cdot 11$
REFRAC REFRAC BASE VELOC INTERC MENT $\mathrm{KM} / \mathrm{SEC}$ SEC

$1.79 \quad 4.50 \quad 1.304$

$2.86 \quad 5.27 \quad 1.363$

117

.16

$3 \cdot 09$

$4 \cdot 28$

$4 \cdot 95$

$6 \cdot 14$

$4 \cdot 44$

$5 \cdot 24$

4.88

$5 \cdot 40$

$5 \cdot 82$

$6 \cdot 43$

$4 \cdot 94$

$5 \cdot 35$

$4 \cdot 20$

4.8 ?

$2 \cdot 88$
$3 \cdot 30$

.167

.801

4.10 1.515

$1.93 \quad 2.277$

$3 \cdot 10 \quad 4 \cdot 020$

$4 \cdot 30 \quad 5.559$

$4.80 \quad 5.657$

$1 \cdot 90 \quad 2.567$

$3 \cdot 60 \quad 3.929$

$3.50 \quad 5.518$

$1 \cdot 91 \quad 3.044$

$2 \cdot 40 \quad 4 \cdot 357$

$\begin{array}{ll}3.80 & 5.961 \\ 4.40 & 6.191\end{array}$

$3 \cdot 19 \quad 5.912$

$4.50 \quad 6.299$

$6.20 \quad 7.107$

$4 \cdot 20 \quad 6.838$

$5.10 \quad 7.333 \quad 1$

$2 \cdot 86 \quad 6.355$

$5.00 \quad 7.836$

$5.10^{\circ} \quad 7.407 \quad 1$

$5.40 \quad 6.795 \quad 1$

$3.00 \quad 6.106$

$4.20 \quad 6.724 \quad 1$

$3.71 \quad 6.241$

$5.44 \quad 7.028 \quad 1$

$3 \cdot 10 \quad 6.262 \quad 1$

$2.50 \quad 6.453$ 
SB SHIP \& LATITUDE LONGITUDE COLRSE TRAVEL LA \# CRUISE DECIMAL DEGREES IN TIME

227 A2 $75+31.067-024.888 \quad 066$

229 A2 $75+32.617-023.385 .029$

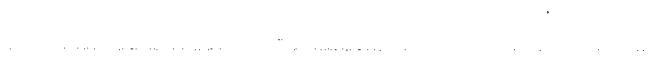

$230 \mathrm{A2} \quad 75+33.500 \quad-022 \cdot 782 \quad \mathrm{c} 29$

$\begin{array}{llllll}231 & A_{2} & 75 & +34.675 & -021.987 & 107\end{array}$

232 A2 $75+34.100 \quad-020 \cdot 285 \quad 109$

$234 \mathrm{AL} 75+33.572=018.703 \quad 113$

237 A2 $75+32.505 \quad .015 .630 \quad 114$

$23^{8} A_{2} \quad 75+31.948 \quad-014 \cdot 178 \quad 10^{9}$

$393_{2} 75 \quad+31.850 \quad-013.913 \quad 10^{5}$

.

240 A2 $75+31.463-012.540 \quad 107$

240 A2 $75 .+31.463 .012 .540107$

$241 A 275+31.027 \cdot 011 \cdot 263116$

$\begin{array}{llllll} & & & & 4.532 \\ & 4.920 \\ & & \\ 243 \quad 218 \\ 3.357\end{array}$

PAGE 17

7.138

7.178

7.477

7.162

7.405

$7 \cdot 787$

8.100

8.400

7.130

$7 \cdot 403$

7.614

6.896

7.530

$7 \cdot 918$

6.854

$7 \cdot 225$
$5 \cdot 218$

6.103

6.243

$6 \cdot 270$
4.583

4.846

5.509

5.718

$6 \cdot 348$

$6 \cdot 843$

6.457

6.731

7.169

5.468

5.704

6.236

6.586

6.931

7. 308

4.591

5.095

5.421

6.330

3.043

3.625

4.183

EASTERN ATLANTIC
LAYER LAYER STD DEV LAYER

DIP VELOC VELOC THICK DEG KM/SEC KM/SEC KM

TOTAL

THICK KM

5.4

$7 \cdot 347-2 \cdot 6$

$8.010-2.6 \quad 3.00 \div .00$

$1 \cdot 0$

1.9

$1 \cdot 52$

.01

$2 \cdot 36 \quad .44$

$1.52 \quad .00$

$.14 \quad .24$

. $2.57 \quad 1.25$

$.0 .88+.00$

$.0 .20+.00$

$\begin{array}{lll}.0 & 1.52 & .00\end{array}$

.7

1.57

$1 \cdot 67$

- 23

$-3 \cdot 2$

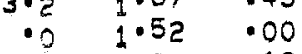

.45

- $0 \quad 1.86 \quad .19$

$\begin{array}{rll}18.0 & 2.77 & 1.23 \\ 4.31 & 2.62\end{array}$

$\begin{array}{lll}1.31 & 2.62\end{array}$

$-7.2 \quad 3.18 \quad 4.10$

$.6 \quad 1.51 \quad .00$

$.0 \quad 1.65 \quad .15$

$\begin{array}{rll}-3.1 & 2.04 \quad .11\end{array}$

$.3 .1 \quad 2.70 * .00$

$\begin{array}{lll}.8 & 1.51 & .01 \\ 1.1 & 1.93 & .49\end{array}$

$5 \cdot 1$

$-2$

$-1.0$

$2 \cdot 5$

$2 \cdot 12$

$1 \cdot 51$

$2 \cdot 10$
2.62
1.51

1.50

2.30

$5 \cdot 41$

$5 \cdot 70$

.996 .69

$5.44 \quad 5.44$

.35

5.43

.26

.49

.60

.78

5.41

.23

.20

$5 \cdot 22$

.28

.46

.84

$5 \cdot 19$

.59

$4 \cdot 39$

.24

.14

3.45

.25

.70
4.32

.65

$4 \cdot 32$

.26

.45

.34

.65

$.3 \quad 2.99 \quad .32$

1.29

$\begin{array}{lll}.3 & 2.48 & .00\end{array}$

$1 \cdot 0$

$1 \div 0$

$\begin{array}{ll}1.51 & .00 \\ 1.69 & .15\end{array}$

.00

$1: 0$

1.77

.12

$1.0 .06+.00$

$10.8 \quad 5.00+.00$

$\because 1.50 \quad .00$

$.0 \quad 1.75 \quad .73$

$\begin{array}{lll}0 & 0.26 & .26\end{array}$

$\begin{array}{lll}-0 & 2.78 & 1.30\end{array}$

$\begin{array}{lll}.0 & 2.98 & 1.306\end{array}$

- $8 \quad 1.50 \quad .00$

$\begin{array}{lll}.8 & 1.88 & .21 \\ .8 & 2.38 & 1.27\end{array}$

$\begin{array}{lll}.8 & 2.38 & 1.27 \\ .8 & 2.57 & .83\end{array}$

$\begin{array}{lll}.6 & 1.50 & .01 \\ .6 & 1.77 & .51\end{array}$

$4 \cdot 12$

- 20

.49

.23

.82

3.30

18

.18
.57

.45

$1 \cdot 36$

$2 \cdot 29$

.55

.66

.45
.63

$2 \cdot 12$

.48

5.80

$5 \cdot 43$

5.69

$6 \cdot 18$

$6 \cdot 78$

$7 \cdot 56$

$5 \cdot 41$

$5 \cdot 63$

5.83

$5 \cdot 22$

$5 \cdot 51$

5.97

6.80

5.78

$4 \cdot 39$

4.63

4.77

4.81
3.45

3.70

$4 \cdot 41$

$4 \cdot 32$

4.98

5.63

$4 \cdot 32$

$4 \cdot 57$

$5 \cdot 02$

$5 \cdot 37$

6.02

$4 \cdot 12$

$4 \cdot 32$

$4 \cdot 80$

$5 \cdot 29$

$5 \cdot 52$

$6 \cdot 34$

$3 \cdot 30$

3.48

$4 \cdot 05$

4.50

$5 \cdot 86$

2.29

?. 33

$4 \cdot 58$

$2 \cdot 12$
$2 \cdot 60$
REFRAC REFRAC BASE

VELAC INTERC MENT

$\mathrm{KM} / \mathrm{SEC}$ SEC

$.8 \quad 3.20 * .00$

$3 \cdot 95$

$3 \cdot 00$

$2.88 \quad 6.205$

$5 \cdot 50 \quad 7.133 \quad 1$

$2 \cdot 42 \quad 5.928$

$3.88 \quad 7.008$

$5 \cdot 20 \quad 7.677$

$6 \cdot 18$

8.181

$4.50 \quad 7.721 \quad 1$

$4 \cdot 36 \quad 6.845$

1

$3 \cdot 60 \quad 6.215$

$7 \cdot 20 \quad 5 \cdot 324 \quad 1$

$5.90 \quad 6.220 \quad 1$

1

$2.86 \quad 5.265$

$4.06 \quad 6.227$

$5.00 \quad 6.507$

$7 \cdot 20 \quad 6.829$

1

$2 \cdot 60 \quad 4 \cdot 051$

$4 \cdot 05$

5.545

$2 \cdot 36 \quad 2 \cdot 723$

$2 \cdot 99 \quad 3 \cdot 304$

$4 \cdot 20 \quad 4.459$

$2.57 \quad 2.752$

R/V ATLANTIS II 67 AND 75 
SB SHIP \& LATITUDE LONGITUOE COURSE TRAVEL LAYER LAYER STD DEV LAYER \# CRUISE DECIMAL DEGREES IN $(-)$ SEUTH (*) DESST DEG

$244 A_{2} 75+29.505-012.085 \quad 139$

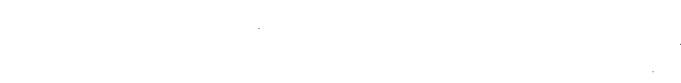

245 A2 $75+28.757-011.460 \quad 138$

$246 A_{2} \quad 75+28.612 \cdot 011.650 \quad 772$

247 AP $75+28.607-011.960 \quad 280$

248 A2 $75+28.695 \quad-012.698 \quad 285$

$249 \mathrm{AC} 75+28.215 \quad-013.672 \quad 172$

$250 \mathrm{A2} 75+27.262 \quad-013.667 \quad 310$

$251 \mathrm{AE} \quad 75 \quad+27.575 \quad-014.160 \quad 314$

$252 A Z, 75+28.358 \quad-015 \cdot 053 \quad 316$

$253 A_{2} 75+28.665-015.418 \quad 317$

254 A2 $75+29.123 \quad 016.047 \quad 314$

FAGE 18
TIME DIE VELQC VELOC THICK THICK SECS DEQ KM/SEC KM/SEC KM KM

4.638

$4 \cdot 890$

2. 645

3.012

$3 \cdot 411$

$3 \cdot 755$

$4 \cdot 340$

1100

.150

.440

.570

.880

.320

.860

- 93

.100

.180

- 230

.500

.373

- 670

- 910

$1 \cdot 160$

$1 \cdot 290$

$1 \cdot 928$

2.094

2.419

2.765

$3 \cdot 369$

.470

.710

$1 \cdot 120$

$2 \cdot 641$

2.936

3.156

3.884

4.182

4.560

4.220

4.474

4.595

$4 \cdot 915$

4.306

5.243
6.179

6.719
4.875

4.875
5.188

5.720

6.248
.6 .57 .53

$\begin{array}{lll}.6 & 4.204 & .00 \\ .5 & 1.50 & .01\end{array}$

- $5 \quad 1 \cdot 91$

.52 .09

. 5.33

.52 .84

$-5$

10

-

. 0

.0

- 0

.0

.1

$\cdots 1$

$\because 1$

.11
. .5
.05

.5
.05
. .5

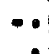

-

.

.

$-\because \frac{3}{2}$

$-4 \cdot 3$

$-4 \cdot 3$

$-4 \cdot 3$

$\ldots$

-14
-04

$\ldots$

$\because 4 \quad 2.80$

.04
.05

$-.5$

$-1 \cdot 7$

$-4 \cdot 4$

4.

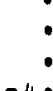

.0
.0

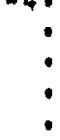

.71

$3 \cdot 30+.00$

1.51 .00

2.00* .00

$2.56+.00$

$3.57+.00$

$4.00+.00$

$1.52 \quad .00$

$2.00 * .00$

$3.06+.00$

$1.52 \quad .00$

$2.53+.00$

$3.06+.00$

$3 \cdot 27+.00$

$1.51 \quad .00$

$1.77+.00$

$2.18+.00$

$2.62+.00$

$3 \cdot 11+.00$

$1.50 \quad .01$

1.89

1.97

$1 \cdot 81$

$2 \cdot 24$

$1 \cdot 51$

$1.60 *$

$1.70+.00$

$1.50 \quad .01$

1.51

$2 \cdot 35$

$3 \cdot 20$ *

$1 \cdot 50$

$2 \cdot 20$

2. 33

द. 58

1.5

$2 \cdot 04$

$? \cdot 28$

4.40
1.50

4.50
1.72

$2 \cdot 27$
$2 \cdot 49$

\subsection{3} 1.73

.32

.38

$2.00 * .00$
REFRAC REFRAC BASE

VELAC INTERC MENT $K M$ KEC SEC

$4 \cdot 20 \quad 4 \cdot 145$

$7 \cdot 20 \quad 6.165$

$2 \cdot 58 \quad 2 \cdot 332$

$3 \cdot 30 \quad 3 \cdot 155$

$5.00 \quad 4.171$

$2.56 \quad .131$

$3.57 \quad .405$

$4.33 \quad .691$

$3.06 \quad .271$

$4.64 \quad .815$

$2.53 \quad .088$

$3.06 \quad .208$

$3.27 \quad .305$

$4.87 \quad .889$

$1.77 \quad 185$

$2.18 \quad .510$

$2.62 \quad .869$

$3 \cdot 11 \quad 1.314$

$3 \cdot 47 \quad 1.593$

$2.52 \quad 2.095$

$3.07 \quad 2.670$

$1 \cdot 70 \quad \cdot 394$

$1.90 \quad .859$

$2 \cdot 27 \quad 2 \cdot 330$

$2 \cdot 70 \quad 3 \cdot 164$

$3 \cdot 80 \quad 4 \cdot 108$

$3.00 \quad 4.461$

1

$2 \cdot 42 \quad 4.107$

$2 \cdot 53 \quad 4 \cdot 344$

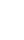

$4 \cdot 22$

$4 \cdot 74$

1.73

$2 \cdot 06$

2.44

2.91

$3 \cdot 39$

$4 \cdot 35$

. 08

. 13

. 54

.78
1.09

.07

- 31

1.14

1.14
.07

- OR

.24
.40

- 40

$1 \cdot 37$

. 28

.59

.98

$1 \cdot 56$

$1 \cdot 97$

$1 \cdot 45$

$1 \cdot 61$

$1 \cdot 93$

2.24

$2 \cdot 9 ?$

.54
.97

1.98

2. 21

$2 \cdot 4$ ?

$3 \cdot 28$

3.69

$4 \cdot 14$

$3 \cdot 17$

$5 \cdot 45$

5.59

6.01

$3.5 ?$

$4 \cdot 06$

$5 \cdot 13$

6.32

$3 \cdot 67$

3.94

$4 \cdot 54$

5.2 ?
$4.00 \quad .538$

R/V ATLANTIS II 67 AND. 75 

SE SHIP \& LATITUDE LONGITUDE COLRSE TRAVEL LAYER LAYER STD OEV LAYER TO
\# CRUISE DECIMAL DEGREES IN TIME DIP VELOC VELOC THICK $(-)=S Q U T H(-) \times W E S T$ DEG SECS

255 A2 $75+29.905-017.023 \quad 317$

256 A2 $75+30.838 \quad-018 \cdot 108 \quad 058$

257 A2 $75+31.527-016.883 \quad 124$

259 A2 $75+30.752 \quad-015.518 \quad 124$

$260 \mathrm{A2} 75+30.298=014.757 \quad 122$

$261 A 2 \quad 75+29.743-013.587 \quad 120$

$262 A 275+29.398-012.827 \quad 124$

263 A2 $75+28.952 \quad .012 .028 \quad 122$

264 A2 $75+30.527 \quad-012 \cdot 082 \quad 0^{43}$

265 A2 $75+30 \cdot 275-011 \cdot 732 \quad 162$

266 A2 $75+31.857 \quad-010.068 \quad 067$

267 A2 $75+31.888-010.022 \quad 302$

268 A2 $75+32 \cdot 428 \cdot 011 \cdot 232 \quad 306$

FAGE 19

\section{5.}

$\begin{array}{lr}5.403 & - \\ 5.993 & \\ 6.362 & -1 \\ 6.942 & -12 \\ 6.111 & \end{array}$

6.831

$8.072 \cdot 10 \cdot 0$

5.919

6.601

$7 \cdot 355-1 \cdot 7$

$5 \cdot 223$

$6 \cdot 135$

7.084

3.973

$4 \cdot 271$

$5 \cdot 346$.

$6.200-11.5$

3.635
3.972

4.179

$4 \cdot 525$

$5 \cdot 185$

$2 \cdot 247$

2. 883

3.189

3.642

.172

172
.380

.480

.940

1.810

2.551

3.065

$3 \cdot 552$

$4 \cdot 312$

$3 \cdot 033$

$3 \cdot 563$

4.067

$4 \cdot 496$

.161

.400

1.020

1.680

- 180

$1 \cdot 310$

$2 \cdot 150$

4.528

5.082
$7.106 \quad-2.7$

DEG KM
$\mathrm{KM}$

KM/SEC KM/SEC

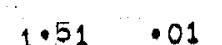

$\begin{array}{ll}1.51 & .01 \\ 1.81 & .08\end{array}$

$2.32 \quad 1.00$

$3 \cdot 73 \quad 1.16$

1.51 .00

$1.73 \quad .14$

$\begin{array}{ll}1 \cdot 73 & 114 \\ 3 \cdot 24 & 33\end{array}$

$3.58 \quad 1.74$

$\begin{array}{ll}3 \cdot 58 & 1 \cdot 74 \\ 1 \cdot 51 & .01\end{array}$

$2.13 \quad .14$

$2 \cdot 28 \cdot 41$

$3.66 \quad 1.53$

$1 \cdot 8$

$1 \cdot 8$

.68

$\begin{array}{lll}1.8 & 2 \cdot 42 & 118 \\ 3.1 & 3.10 & 1.62\end{array}$

$1.8 \quad 1.50 \quad .00$

.5

1.50

$3 \cdot 07$

.18

2.99 .25

$3.20+.00$

1.50 .01

$\begin{array}{ll}1.71 & .12 \\ 1.96 & .49\end{array}$

$2 \cdot 15.21$

$\begin{array}{lll}2.5 & 2.15 & .21 \\ 2.5 & 2.82 & 2.43\end{array}$

$\begin{array}{rrr}.0 & 1.50 & .02 \\ 0 & 1.93 & 16\end{array}$

$\begin{array}{lll}.0 & 1.93 & .16 \\ .0 & 2.14 & .24\end{array}$

$\begin{array}{lll}-2 \cdot 4 & 2.58 & .36 \\ -2.4 & 3.07 & .08\end{array}$

$.0 \quad 1.51 .00$

$2.11+.00$

$2.68+.00$

$3.84+.00$

$1.50 \quad .01$

$\begin{array}{ll}1.50 & .01 \\ 1.91 & .17 \\ 2.69 & .87\end{array}$

2.69
$3.50+.00$

$1.50+.01$

$\begin{array}{ll}2 \cdot 02 & .05 \\ 2 \cdot 32 & .15\end{array}$

$3 \cdot 33 \cdot 24$

$1.51 \quad .00$

$2.25+.00$

$2.58+.00$

$3.43+.00$

1.51 .00

$2.42+.00$

$3 \cdot 22+.00$

$1.51 \quad .01$

$\begin{array}{ll}1.78 & .01 \\ 1.28\end{array}$
TOTAL.

THICK.

KM

REFRAC REFRAC BASE

VELAC INTERC MENT

KMISEC SEC

$4.07 \quad 4.07$

$.53 \quad 4.51$

.434 .93

$1.08 \quad 6.01$

$4.62 \quad 4.62$

$.63 \quad 5.24$

$1.14 \quad 6.39$

.96 .7 .34

$4.47 \quad 4.47$

.73

.58

.45

3.94

.76

.49

.84

2.99

.31

1.61

1.44

$5 \cdot 20$

$5 \cdot 77$

$6 \cdot 23$

$3 \cdot 94$

$4 \cdot 70$

$5 \cdot 19$

6.03

$3 \cdot 30$

$4 \cdot 91$

$6 \cdot 35$

2.73

$3 \cdot 0$ ?

$3 \cdot 2$ ?

3.60

4.53

1.69

$2 \cdot 30$

$2 \cdot 63$

$3 \cdot 2$ ?

$4 \cdot 46$

1.09

$1 \cdot 75$

$2 \cdot 41$

$3 \cdot 07$

$3 \cdot 58$
$3 \cdot 58$
$3 \cdot 58$

3.40

$4 \cdot 12$

3.48
$4 \cdot 10 \quad 6 \cdot 448 \quad 1$

$4.00 \quad 6.724$

$6.60 \quad 8.475 .1$

$3 \cdot 73 \quad 6 \cdot 784$

2.30 4.401

$3.17 \quad 5.560$

$5.60 \quad 6.636$

$3 \cdot 20 \quad 4.768$

$4 \cdot 10 \quad 5.452$

$1.96 \quad 2.490$

$2.70 \quad 3.635$

$4.60 \quad 4.992$

$-$

$2.42 \quad 2.439$

$4.40 \quad 4.116$
- 13

.35

.47

$1 \cdot 9 ?$

$2 \cdot 82$

4. 12

.39

$1 \cdot 19$

$2 \cdot 3$ ?

114

$1 \cdot 50$

$2 \cdot 84$

$3 \cdot 89$

$2 \cdot 11 \quad .146$

2.37 $\cdot 323$

$2.68 \quad .426$

$3.85 \quad .901$

$6 \cdot 23$

1.789

1

$3 \cdot 50 \quad 3.103$

$4 \cdot 00$

3.949

$2.60 \quad 2.977$

$3.69 \quad 3.862$

$2 \cdot 25 \quad .155$

$2.58 \quad .360$

$3.43 \quad .952$

$5.51 \quad 1.649 \quad 1$

$2.42 \quad 168$

$3.22 \quad 1.181$

$4.41 \quad 2.037$

1

R/V ATLANTIS II 67 AND 75 


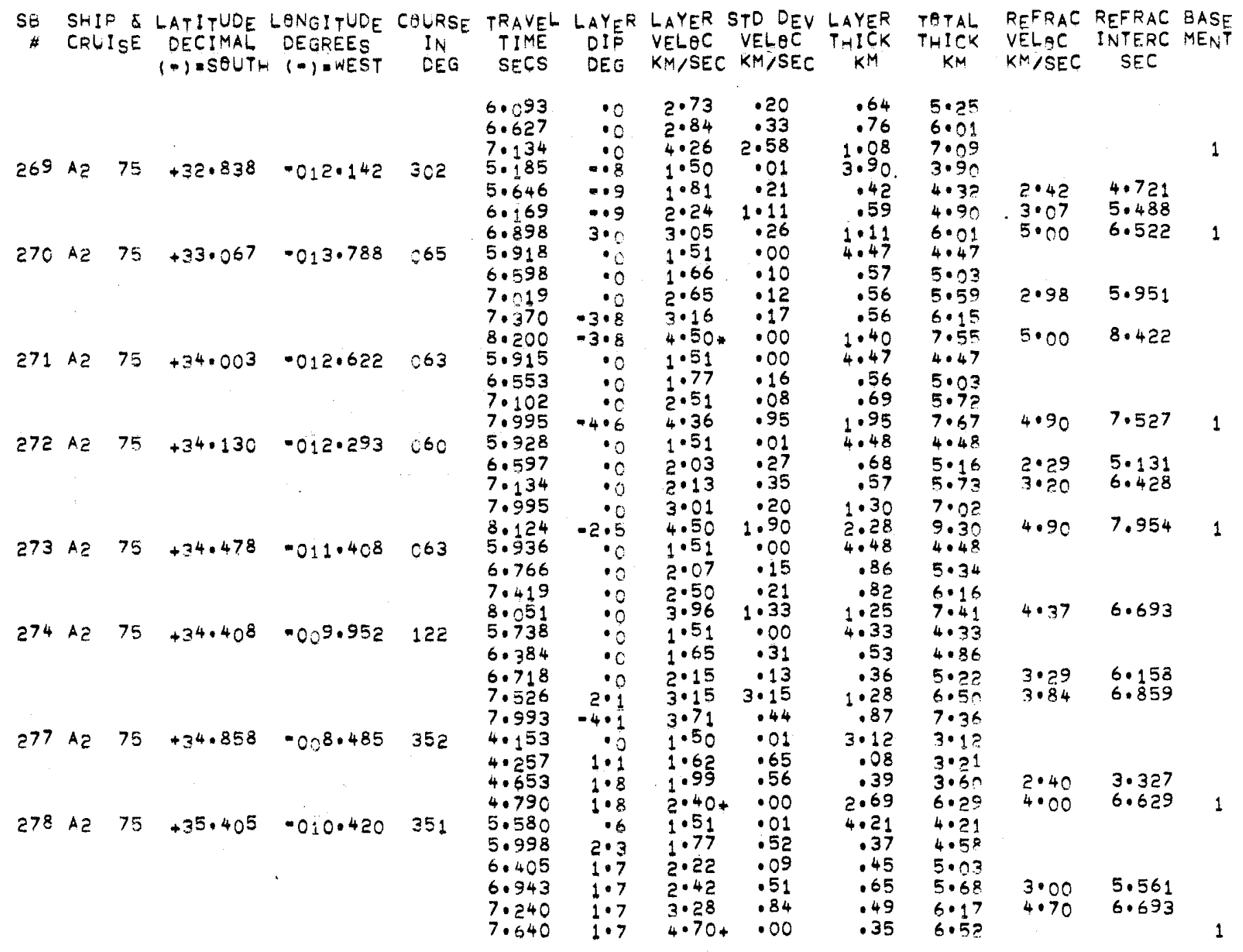




\section{ACKNOWLEDGMENTS}

S. T. Knott carefully reviewed the analytic procedure which led to several improvements. K. E. Prada designed and implemented the computer system. His excellent collection of utility programs was most effective in program development. Elazar Uchupi and K. O. Emery interpreted the seismic profiles and collated the observations from the cruises, enabling us to weigh these determinations against their geological settings. The continuity in. recording procedure and logging provided by the three watch chiefs on board for the entire cruise expedited the analysis. They were R. Davis, D. Hathaway, C. Hornig, C. Kenah, D. Shaughnessy, R. Steward, and W. Wenk.

Grateful acknowledgment is made to the officers and crew of the $R / V$ ATLANTIS II, especially to their conscientious responses to the query, "Will traffic permit us to maintain course and speed for the next three hours and will any ship run down the buoy if we launch it here?"

The procedure for analysis was developed under contracts with code 480 of the Office of Naval Research (Knott and Hoskins, in preparation). NSF grant GA-27516 supported further refinements. The data gathering and processing was done under NSF-IDOE grant GX-28193. 


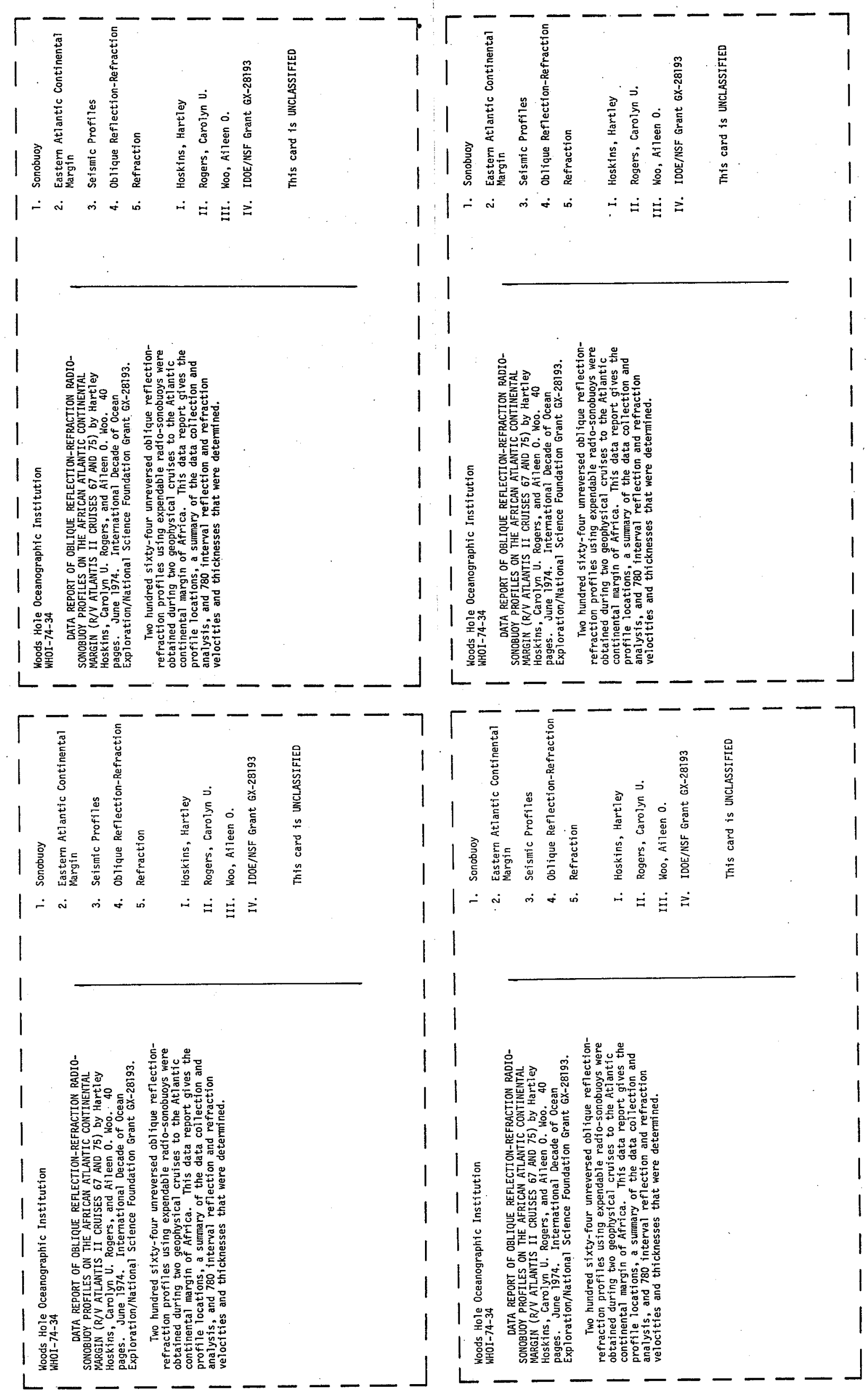




\begin{tabular}{|c|c|c|}
\hline $\begin{array}{l}\text { BIBLIOGRAPHIC DATA } \\
\text { SHEET }\end{array}$ & 1. Report No. & 3. Recipient's Accession No. \\
\hline \multirow{2}{*}{\multicolumn{2}{|c|}{$\begin{array}{l}\text { 4. Title and Subtitle } \\
\text { DATA REPORT OF OBLIQUE REFLECTION-REFRACTION RADIO-SONOBUOY } \\
\text { PROFILES ON THE AFRICAN ATLANTIC CONTINENTAL MARGIN (R/V } \\
\text { ATLANTIS II CRUISES } 67 \text { AND 75) }\end{array}$}} & $\begin{array}{l}\text { 5. Report Date } \\
\text { June } 1974\end{array}$ \\
\hline & & 6. \\
\hline \multicolumn{2}{|c|}{$\begin{array}{l}\text { 7. Author(s) } \\
\text { Hartley Hoskins, Carolyn U. Rogers, and Aileen } 0 . \text { Woo }\end{array}$} & $\begin{array}{l}\text { 8. Performing Organization Rept. } \\
\text { No. WHOI }-74-34\end{array}$ \\
\hline \multirow{2}{*}{\multicolumn{2}{|c|}{$\begin{array}{l}\text { 9. Performing Organization Name and Address } \\
\text { Woods Hole Oceanographic Institution } \\
\text { Woods Hole, MA } 02543\end{array}$}} & 10. Project/Task/Work Unit No. \\
\hline & & $\begin{array}{l}\text { 11. Contract/Grant No. } \\
\text { IDOE/NSF } 28193\end{array}$ \\
\hline \multirow{2}{*}{\multicolumn{2}{|c|}{$\begin{array}{l}\text { 12. Sponsoring Organization Name and Address } \\
\text { National Science Foundation/International Decade of Ocean } \\
\text { Exploration Grant GX-28193 }\end{array}$}} & $\begin{array}{l}\text { 13. Type of Report \& Period } \\
\text { Covered } \\
\text { Technical }\end{array}$ \\
\hline & & 14. \\
\hline
\end{tabular}

15. Supplementary Notes

16. Abstracts

Two hundred sixty-four unreversed oblique reflection-refraction profiles using expendable radio-sonobuoys were obtained during two geophysical cruises to the Atlantic continental margin of Africa. This data report gives the profile locations, a summary of the data collection and analys is, and 780 interval reflection and refraction velocities and thicknesses that were determined.

17. Key Words and Document Analysis. 17a. Descriptors

1. Sonobuoy

2. Eastern Atlantic Continental Margin

3. Seismic Profiles

4. Oblique Reflection-Refraction

5. Refraction

17b. Identifiers/Open-Ended Terms

17c. COSATI Field/Group

18. Availability Statement 